\title{
High velocity anomaly beneath the Deccan volcanic province: Evidence from seismic tomography
}

\author{
H M IYER*, V K GAUR, S S RAI, D S RAMESH, C V R RAO, \\ D SRINAGESH and K SURYAPRAKASAM \\ National Geophysical Research Institute, Hyderabad 500007, India \\ \# US Geological Survey, Menlo Park CA, USA
}

\begin{abstract}
Analysis of teleseismic $P$-wave residuals observed at 15 seismograph stations operated in the Deccan volcanic province (DVP) in west central India points to the existence of a large, deep anomalous region in the upper mantle where the velocity is a few per cent higher than in the surrounding region. The seismic stations were operated in three deployments together with a reference station on precambrian granite at Hyderabad and another common station at Poona. The first group of stations lay along a west-northwesterly profile from Hyderabad through Poona to Bhatsa. The second group roughly formed an L-shaped profile from Poona to Hyderabad through Dharwar and Hospet. The third group of stations lay along a northwesterly profile from Hyderabad to Dhule through Aurangabad and Latur. Relative residuals computed with respect to Hyderabad at all the stations showed two basic features: a large almost linear variation from approximately $+1 \mathrm{~s}$ for telescisms from the north to $-1 \mathrm{~s}$ for those from the southeast at the western stations, and persistance of the pattern with diminishing magnitudes towards the east. Preliminary ray-plotting and three-dimensional inversion of the $P$-wave residual data delineate the presence of a $600 \mathrm{~km}$ long approximately $\mathrm{N}-\mathrm{S}$ trending anomalous region of high velocity $(1-4 \%$ contrast) from a depth of about $100 \mathrm{~km}$ in the upper mantle encompassing almost the whole width of the DVP. Inversion of $P$-wave relative residuals reveal the existence of two prominent features beneath the DVP. The first is a thick high velocity zone $(1-4 \%$ faster) extending from a depth of about $100 \mathrm{~km}$ directly beneath most of the DVP. The second feature is a prominent low velocity region which coincides with the westernmost part of the DVP. A possible explanation for the observed coherent high velocity anomaly is that it forms the root of the lithosphere which coherently translates with the continents during plate motions, an architecture characteristic of precambrian shields. The low velocity zone appears to be related to the rift systems (anomaly $28,65 \mathrm{Ma}$ ) which provided the channel for the outpouring of Deccan basalts at the close of the Cretaceous period.
\end{abstract}

Keywords. Deccan volcanic province; seismic tomography; deep structure; high velocity anomaly; teleseismic residuals; three-dimensional inversion.

\section{Introduction}

The Deccan volcanic province (DVP) encompasses an area of about a half million $\mathrm{km}^{2}$ in west-central India with a possible extension of an additional 1 million $\mathrm{km}^{2}$ beneath the Arabian Sea to the west. The Deccan basalts are primarily of tholeiitic composition with a few pockets of acidic volcanism. The volcanic flows occur as a relatively thin veneer at the surface with a thickness of $1-1.5 \mathrm{~km}$ in the western third of the province, thinning to about $0.4 \mathrm{~km}$ in the middle section and to a few meters in the eastern section (Kaila 1982). The bulk of the Deccan volcanism is now known to have occurred around $65 \mathrm{Ma}$ (Mahoney 1988). About $200 \mathrm{Ma}$ ago, India was wedged in the southern latitudes between Africa and Antarctica as part of the 
super-continent Gondwanaland. During the Triassic period,-it split off and started migrating northwards as an independent continent eventually colliding with Eurasia about $45 \mathrm{Ma}$ ago. Morgan (1972) proposed that the Deccan basalts erupted during this northward migration as the sub-continent traversed over a mantle plume associated with the Reunion hot spot.

\subsection{Gravity}

The Deccan Traps lie in a broad gravity low which encompasses the whole of the south Indian shield. Within this broad low are shorter wavelength "lows" and "highs". A few of these short wavelength features have been interpreted variously: uplift and subsidence due to cycles of volcanic activity (Kailasam 1975); crustal intrusion of mafic rock (Takin 1966) and linear channels of magma flow (Qureshy 1981). We also show later that preliminary two-dimensional modelling of the gravity data indicates a lower-than-normal density in the upper mantle beneath the central part of the DVP.

\subsection{Deep seismic sounding}

Deep seismic sounding was carried out along two profiles over the Koyna region of the Deccan Traps and several others in the Saurashtra Peninsula and across the Narmada-Son lineament (Kaila et al 1981, 1985; Kaila 1982). Though the velocity models obtained from these profiles reflect the thickness of the surficial basalts up to $2 \mathrm{~km}$, the crustal structure beneath the DVP is not significantly different from that of the precambrian south Indian shield model elsewhere as revealed by data along the Kavali-Udipi profile (Kaila 1982). Moho depth is found to vary from $39-40 \mathrm{~km}$ west of Koyna to $36-38 \mathrm{~km}$ eastwards. There is no evidence of drastic changes in the depth of major discontinuities like the Conrad discontinuity across the profile nor is there evidence of unusually high upper or lower crustal velocities which are characteristic of rift zones.

\subsection{Heat-flow}

Except for the Cambay graben where the heat-flow is somewhat higher-than-normal, other parts of the Deccan Traps show normal heat flow typical of stable continental regions (Gupta and Gaur 1984). Except for implying that the volcanic episode responsible for the Deccan volcanic flows did not leave a detectable thermal remanance in the lithosphere, the heat-flow data do not provide any information on the deep structure beneath the DVP.

In summary, the available geophysical data are inadequate to constrain a source model for the Deccan volcanism. For example, if the magmatism was caused by a mantle plume, extensive melting, differentiation and variations in physical and chemical properties of the deep structure of the lithosphere might have taken place. But the available heat-flow and deep seismic sounding data are inadequate to model the structure of the lithosphicie and asthenosphere beneath the DVP which would expose alterations in structure associated with that volcanism. With this background, we designed a tomographic experiment to model in three dimensions the compressional wave velocity structure of the upper mantle beneath the DVP. Rusults from this experiment reveal a vast anomalous region in the upper mantle of the 
DVP where the $P$-wave velocity is a few per cent higher than in the surrounding region. This is the first effort to map the deep structure beneath the DVP.

\section{Tomography using teleseismic residuals}

Teleseismic residuals provide an excellent approach to model the two- and threedimensional velocity structures of the earth's crust and mantle. Numerous publications describe the use of the technique to delineate heterogeneous earth structure from global and continental scales to local scale. Recent introduction of inversion methods for teleseismic residuals (also called seismic tomography) has considerably enhanced the power of the teleseismic residual technique (Aki et al 1977; Humphreys and Clayton 1988). Tomographic modelling of small and large heterogeneities in the earth's crust and mantle have been carried out in diverse geologic settings (Thurber and Aki 1987; lyer and Hitchcock 1988). The present study is the first application of this technique in India.

\section{Estimation of teleseismic residuals}

Teleseisms (earthquakes occurring at distances greater than approximately 20 degrees from the recording station) are recorded by a dense array of seismic stations sited over the region of interest to provide the data for tomography. The average instrument spacing in the array limits the smallest size of the velocity anomaly that can be resolved. Two-dimensional arrays yield three-dimensional models, while linear arrays yield two-dimensional models. Arrival times of the first seismic phases (P or PKIKP, PKP) are read from the seismograms and travel time from the source to the recording stations are estimated from a knowledge of the earthquake origin times. Knowing the hypocentral location of the earthquake, theoretical travel times are computed using a standard seismological table. In the present study, we used the $\boldsymbol{P}$-wave travel time tables of Herrin (1968a). The travel-time residuals are simply the differences between the observed and theoretical travel times. In the mathematical notation we write,

$$
R_{i j}=T_{i j}^{o}-T_{i j}^{\text {th }}
$$

where, $\boldsymbol{R}_{i j}$ is the absolute residual in seconds, and $T_{i j}$ and $T_{i j}^{\text {th }}$ are the observed and theoretical travel times respectively at station $i$ for event $j$. In addition to the information on the deep structure beneath the seismic array, $R_{i j}$ contains effects due to errors associated with the location and origin times of the earthquakes travel-time tables, and anomalous structure at the source and along the seismic wave paths. In order to isolate the local structures from extraneous effects, relative residuals are computed independently for each event by subtracting the residual at a reference station which hopefully is located outside the anomaly under investigation from the absolute residuals at each station. Alternatively, a weighted mean of absolute residuals for each event can be used as the reference residual. Thus, the relative residual $R R_{i j}$ for event $j$ can be defined as,

$$
R R_{i j}=R_{i j}-R_{r j}
$$


where the subscript $r$ represents the reference station, or as

$$
R R_{i j}=R_{i j}-R_{j}
$$

where $R_{j}$ represents the weighted mean of $R_{i j}$ using all readings available for the event $j$. In this study all weights were chosen to be equal to unity. Consequently, $R_{j}$ is a simple average of absolute residuals for the whole array.

$R R_{i j}$ contains the information needed to model the three-dimensional relativevelocity structure beneath the seismic array. The magnitudes of the relative residuals indicate the change in velocity within the anomaly; the sign of the residuals being negative for high-velocity anomalies and vice versa. The azimuthal pattern of $R R_{i j}$ gives an idea of the depth of the anomaly. As a rule of thumb, it may be remembered that a $10-\mathrm{km}$ long anomalous ray-path in which the seismic velocity differs from the normal by $10 \%$ produces a residual of about $0 \cdot 2 \mathrm{~s}$.

\section{Three-dimensional inversion}

We have used the three-dimensional inversion technique developed by Aki et al (1977) (now popularly referred to as the $\mathrm{ACH}$ technique) to model the teleseismic residuals. Since the ACH technique is described in detail in various papers (Aki et al 1977; Ellsworth and Koyanagi 1977; Iyer et al 1981; Achauer et al 1986) and also its application (Aki 1982; Thurber and Aki 1987; Iyer and Hitchcock 1988), only a brief outline of the theory will be given here along the lines presented by Acharer et al (1986). In this technique, the volume under the array is divided into layers and each layer into a grid of rectangular blocks. An initial $P$-wave velocity from a known or assumed model is assigned to each layer for ray-tracing purposes. It turns out that the final results are not very sensitive to the choice of this initial velocity model. The incident wave at the bottom of the deepest layer is assumed to be plane. As it travels to the seismograph, it encounters an unknown velocity perturbation in each block. The resultant travel time delays and advances accumulated along the ray path equal the observed residual at the surface. In this treatment, the refraction of the seismic ray due to the velocity perturbation is assumed to be negligible. Thus for each ray, the following linear equation can be generated in a matrix form.

$$
A m=d,
$$

where $A$ is a matrix with calculated unperturbed travel times of ray segments, $m$ is a vector containing the unknown fractional slowness perturbations (slowness is the reciprocal of velocity), and $d$ is a vector containing the travel time residuals. Since $A^{T} A$ is near singular, Aki et al (1977) used the damped least-squares method to obtain the model estimate $\hat{m}$ as,

$$
m=\left(A^{T} A+\theta^{2} I\right)^{-1} A^{T} d,
$$

where $\theta^{2}=\sigma_{d}^{2} / \sigma_{m}^{2}$ is the damping parameter and $\sigma_{d}^{2}$ and $\sigma_{m}^{2}$ are the estimated variances of the data and the (unknown) true model $m$ (Achauer et al 1986). It should be remembered that the estimated model contains only relative velocities, as the inputs are relative residuals.

The main drawback with the block inversion described above (resulting from finite 
data) is that it provides only a 'fuzzy' picture of the real model. The degree of 'fuzziness' depends on the density of instruments deployed in the seismic network, the number and azimuthal distribution of teleseisms used and the block size. Fortunately, the damped-least-squares inversion also produces a resolution matrix and standard errors which enable one to estimate the figure of merit for a computed model. The 'true' model $m$ is related to the 'fuzzy' model $\hat{m}$ by the relation

$$
m=R \hat{m},
$$

where $R$ is the resolution matrix. Each element of the symmetric singular matrix $R$ has a value between -1 and +1 with positive values on the diagonal. Therefore, each row of the matrix can be considered as an average kernel relating all of $m$, the tu ue model, to one block of the estimated 'fuzzy' model. Thus large diagonal elements of the resolution matrix imply that $\dot{m}$ is a good representation of $m$. In general, resolution is good towards the middle of each layer and is rather poor near the edges. It is closely related to the number of ray-hits in the block. The standard errors, derived from the diagonal of the covariance matrix, are indicators of the accuracy of each velocity perturbation. As a rule of thumb, the accuracy of individual perturbation estimates are about $( \pm) 2$ times the standard error for 0.8 resolution and ( \pm ) 3-4 times for 0.6 resolution (Evans 1982). When small blocks are used, the accuracy of the inversion can be assessed by looking at the whole resolution matrix for specific blocks.

\section{The experiment}

We operated fifteen vertical-component seismic stations (Teledyne protacorders with $\mathrm{S}-13,1 \mathrm{~Hz}$, seismometers) in and around the southern half of the DVP (figure 1 , table 1), in three consecutive deployments with Hyderabad (HYB) and Poona (POO) being common to the whole experiment. A total of 168 teleseisms mainly from north, northeast, east and southeast, and in the distance range of 19 to 95 degrees were recorded by this network (table 2). The recorders were operated at their maximum drum speed of $240 \mathrm{~mm} / \mathrm{min}$ and internal clocks synchronized with radio time signals broadcast by the National Physical Laboratory, New Delhi. Considerable care was taken in reading the arrival times of the first phases, thereby achieving a time accuracy of $0.05 \mathrm{~s}$. Visual correlation of seismic waveforms and multiple readings (first-break, peak, zero-crossing, trough etc) were used to ensure a high relative accuracy of picks (figure 2). Despite these precautions substantial signal changes across the array led us to discard about $10 \%$ of the readings (figure 3 ). This as well as the low amplitudes of the signals also contributed to the scatter in data. Even so, the large magnitude of the observed relative-residuals permits us to draw significant conclusions.

\section{Data analysis}

\subsection{Variation of residuals with azimuth and distance}

Figures 4-7 show the relative residuals, with respect to HYB at 13 stations plotted against the respective azimuth of seismic arrivals. We used HYB as the reference 


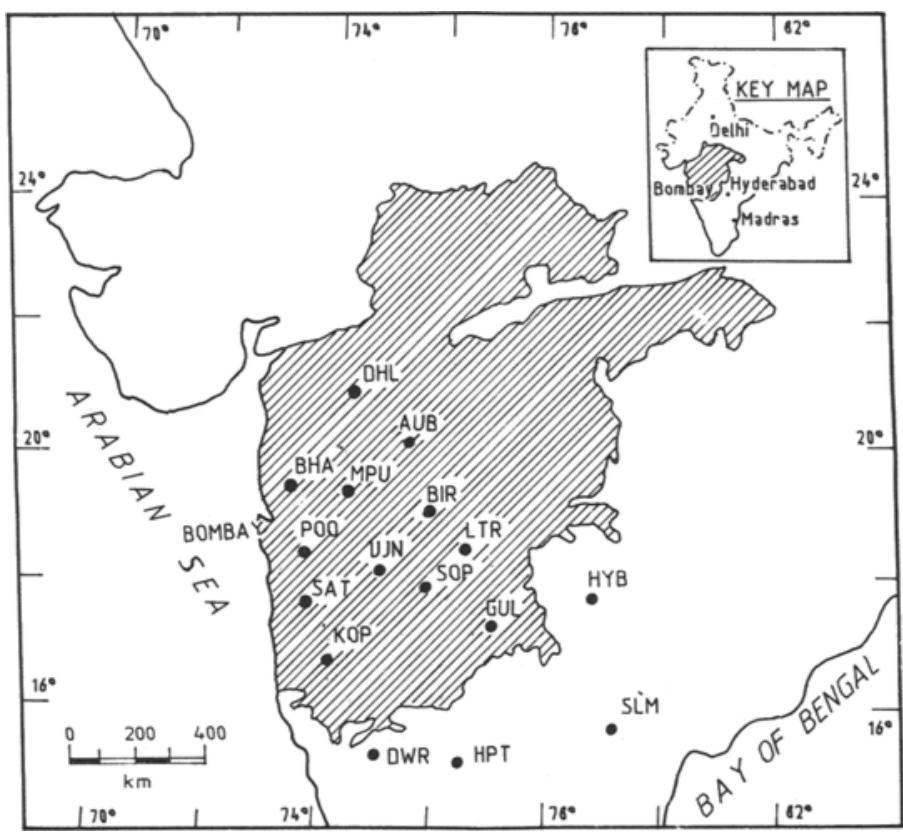

Figure 1. Map of west-central India showing the locations of seismic stations (three-letter names) used in this study. The approximate outline demarking the extension of the DVP.

Table 1. Coordinates of seismic stations.

\begin{tabular}{|c|c|c|c|c|}
\hline Station name & $\begin{array}{l}\text { Latitude } \\
\text { (N) }\end{array}$ & $\begin{array}{l}\text { Longitude } \\
\text { (E) }\end{array}$ & $\begin{array}{l}\text { Elevation } \\
\text { (meters) }\end{array}$ & $\begin{array}{c}\text { Duration of } \\
\text { operation }\end{array}$ \\
\hline Hyderabad (HYB) & $17^{\circ} 25 \cdot 03^{\prime}$ & $78^{\circ} 33 \cdot 18^{\prime}$ & $510-0$ & Sept. 1985-Dec. 1987 \\
\hline Poona (POO) & $18^{\circ} 32 \cdot 84^{\prime}$ & $73^{\circ} 49 \cdot 07^{\prime}$ & $579 \cdot 3$ & Sept. 1985-Dec. 1987 \\
\hline Bhatsa (BHA) & $19^{\circ} 31 \cdot 12^{\prime}$ & $73^{\circ} 24 \cdot 06^{\prime}$ & $185 \cdot 9$ & Dec. 1985 -Apr. 1986 \\
\hline Ujani (UJN) & $18^{\circ} 0400^{\prime}$ & $75^{\circ} 06 \cdot 87^{\prime}$ & 496.9 & Jan. 1986-Apr. 1986 \\
\hline Solapur (SOP) & $17^{\circ} 38.90^{\prime}$ & $75^{\circ} 54.93^{\prime}$ & $475 \cdot 6$ & Jan. 1986-May. 1986 \\
\hline Gulbarga (GUL) & $17^{\circ} 19 \cdot 57^{\prime}$ & $76^{\circ} 53 \cdot 25^{\prime}$ & $470 \cdot 0$ & Jan. 1986-Apr. 1986 \\
\hline Satara (SAT) & $17^{\circ} 40 \cdot 17^{\prime}$ & $74^{\circ} 00+19^{\prime}$ & 670.5 & Jun. 1986-Feb. 1987 \\
\hline Kolhapur (KOP) & $16^{\circ} 40-20$ & $74^{\circ} 15 \cdot 10^{\circ}$ & 563.8 & Jun. 1986-Feb. 1987 \\
\hline Dharwar (DWR) & $15^{\circ} 25 \cdot 27^{\prime}$ & $74^{\circ} 55 \cdot 79^{\prime}$ & $731 \cdot 5$ & Jun. 1986-Jan. 1987 \\
\hline Hospet (HPT) & $15^{\circ} 15 \cdot 31^{\prime}$ & $76^{\circ} 20-58^{\prime}$ & 478.5 & Jun. 1986-Jan. 1987 \\
\hline Srisailam (SLM) & $16^{\circ} 06 \cdot 00$ & $78^{\circ} 53.50^{\prime}$ & $438 \cdot 9$ & Jan. 1987-Mar. 1987 \\
\hline Aurangabad (AUB) & $19^{\circ} 54 \cdot 38^{\prime}$ & $75^{\circ} 18.66^{\prime}$ & $569 \cdot 0$ & May. 1987-Dec. 1987 \\
\hline $\operatorname{Bir}(B I R)$ & $18^{\circ} 58 \cdot 37^{\prime}$ & $75^{\circ} 46 \cdot 28^{\prime}$ & 5600 & May. 1987-Dec. 1987 \\
\hline Latur (LTR) & $18^{\circ} 21 \cdot 25^{\prime}$ & $76^{\circ} 33 \cdot 75^{\prime}$ & 6100 & May. 1987-Dec. 1987 \\
\hline Dhule (DHL) & $20^{\circ} 55.49$ & $74^{\circ} 47.01^{\prime}$ & $265 \cdot 0$ & Jun. $1987-$ Dac. 1987 \\
\hline
\end{tabular}

Table 2. List of teleseisms used in this study which provided readings in at least 3 stations.

\begin{tabular}{|c|c|c|c|c|c|}
\hline \multirow{2}{*}{$\frac{\text { Sl. }}{\text { No. }}$} & \multirow{2}{*}{$\underbrace{\text { Source region }}_{\text {MOLUCAzo(01/03/86) }}$} & \multirow{2}{*}{ Origin time } & \multirow{2}{*}{ - $\begin{array}{c}\text { Depth } \\
(\mathrm{km})\end{array}$} & \multicolumn{2}{|c|}{ Coordinates } \\
\hline & & & & $00: 58 \cdot 32 S$ & $126: 52.08 \mathrm{E}$ \\
\hline 2. & AFGHAN(01/14/86) & $03.03 \cdot 37.4$ & 245 & $36: 20-46 N$ & $71.01 \cdot 44 \mathrm{E}$ \\
\hline 3. & HONSHUza(0I/16/86) & $08 \cdot 34 \cdot 43 \cdot 7$ & 436 & $29: 48 \cdot 12 \mathrm{~N}$ & $138: 39 \cdot 72 \mathrm{E}$ \\
\hline
\end{tabular}


Table 2. (continued)

\begin{tabular}{|c|c|c|c|c|c|}
\hline $\begin{array}{l}\text { Sl. } \\
\text { No. }\end{array}$ & Source region & Origin time & $\begin{array}{c}\text { Depth } \\
(\mathrm{km})\end{array}$ & Coor & inates \\
\hline 4. & MINDROza(01/16/86) & $15 \cdot 45 \cdot 06 \cdot 7$ & 223 & $13: 4 ! \cdot 10 \mathrm{~N}$ & $120: 49 \cdot 32 \mathrm{E}$ \\
\hline 5. & ALUTINtr(01/18/86) & $01 \cdot 59 \cdot 01 \cdot 6$ & 33 & $51: 33 \cdot 18 \mathrm{~N}$ & $173: 06 \cdot 54 \mathrm{~W}$ \\
\hline 6. & MOLUCAtr(01/22/86) & $14 \cdot 57 \cdot 13 \cdot 0$ & 59 & $00: 28 \cdot 02 S$ & $124: 21 \cdot 96 \mathrm{E}$ \\
\hline 7. & HOKADOtr(01/31/86) & $17 \cdot 48 \cdot 04 \cdot 4$ & 69 & $42: 13 \cdot 68 \mathrm{~N}$ & $142: 59 \cdot 76 \mathrm{E}$ \\
\hline 8. & CELBEStr(02/01/86) & $15 \cdot 00 \cdot 35 \cdot 8$ & 392 & $02: 54 \cdot 36 \mathrm{~N}$ & $124: 04 \cdot 50 \mathrm{E}$ \\
\hline 9. & VANATUzc(02/02/86) & $01 \cdot 44 \cdot 05 \cdot 4$ & 31 & $13: 37 \cdot 56 \mathrm{~S}$ & $166: 41 \cdot 58 \mathrm{E}$ \\
\hline 10. & BONINtr(02/03/86) & $20-47 \cdot 35 \cdot 3$ & 508 & $27: 47 \cdot 46 \mathrm{~N}$ & $139: 33 \cdot 12 \mathrm{E}$ \\
\hline 11. & HINDKUzc(02/04/86) & $19 \cdot 43 \cdot 56 \cdot 6$ & 203 & $36: 25 \cdot 56 \mathrm{~N}$ & $70: 41 \cdot 76 \mathrm{E}$ \\
\hline 12. & AFGHANpk(02/11/86) & $07 \cdot 14 \cdot 24 \cdot 2$ & 119 & $36: 22 \cdot 32 \mathrm{~N}$ & $70: 54 \cdot 60 \mathrm{E}$ \\
\hline 13. & HONSHUpk(02/12/86) & $02 \cdot 59 \cdot 30 \cdot 4$ & 30 & $36: 2304 \mathrm{~N}$ & 141:07.68E \\
\hline 14. & PAPNGUza(02/12/86) & $11 \cdot 27 \cdot 45 \cdot 4$ & 34 & $06: 32 \cdot 16 \mathrm{~S}$ & $147: 25 \cdot 98 \mathrm{E}$ \\
\hline 15. & HALMHApk(02/12/86) & $23 \cdot 02 \cdot 12 \cdot 8$ & 135 & $01: 36 \cdot 24 \mathrm{~N}$ & $127: 18 \cdot 30 \mathrm{E}$ \\
\hline 16. & CARSBRzc(02/15/86) & $19 \cdot 56 \cdot 35 \cdot 9$ & 10 & $04: 24 \cdot 72 \mathrm{~N}$ & $62: 42 \cdot 24 \mathrm{E}$ \\
\hline 17. & KURILEpk(02/19/86) & $10 \cdot 54 \cdot 46 \cdot 2$ & 115 & $48: 34 \cdot 74 \mathrm{~N}$ & $153: 24.90 \mathrm{E}$ \\
\hline 18. & PHLPNEzc(02/19/86) & $11 \cdot 40-27 \cdot 5$ & 77 & $18: 56 \cdot 70 \mathrm{~N}$ & $121: 18 \cdot 24 \mathrm{E}$ \\
\hline 19. & AUSTLAZc(02/24/86) & $01 \cdot 26 \cdot 58 \cdot 2$ & 32 & $12: 45 \cdot 24 \mathrm{~S}$ & $114: 36 \cdot 54 \mathrm{E}$ \\
\hline 20. & HALMHApk(02/24/86) & $02 \cdot 31 \cdot 26 \cdot 7$ & 118 & $01: 43 \cdot 74 \mathrm{~N}$ & $127: 21 \cdot 24 \mathrm{E}$ \\
\hline 21. & BANDCApk(03/01/86) & $16 \cdot 41 \cdot 40.6$ & 80 & $06: 18-00 S$ & $130: 56 \cdot 94 \mathrm{E}$ \\
\hline 22. & $\operatorname{KAMCHK}(03 / 02 / 86)$ & $03 \cdot 14 \cdot 41 \cdot 8$ & 118 & $51: 40-68 \mathrm{~N}$ & $156: 56 \cdot 16 \mathrm{E}$ \\
\hline 23. & SOLMONzc(03/06/86) & $12 \cdot 31 \cdot 24 \cdot 0$ & 72 & $07: 00-00 \mathrm{~S}$ & $155: 46 \cdot 86 \mathrm{E}$ \\
\hline 24. & NEWBRTB(03/07/86) & $02 \cdot 46 \cdot 52 \cdot 0$ & 116 & $04: 59 \cdot 40 \mathrm{~S}$ & $151: 42 \cdot 60 \mathrm{E}$ \\
\hline 25. & HANDUKzc(03/11/86) & $23 \cdot 07 \cdot 38 \cdot 2$ & 206 & $36: 28 \cdot 92 \mathrm{~N}$ & $70: 39 \cdot 60 E$ \\
\hline 26. & BONINzc(03/17/86) & $09 \cdot 18 \cdot 25 \cdot 2$ & 476 & $27: 25 \cdot 26 \mathrm{~N}$ & $139: 51 \cdot 96 \mathrm{E}$ \\
\hline 27. & FLOREStr(03/21/86) & $21 \cdot 35 \cdot 35 \cdot 8$ & 614 & $07: 27 \cdot 30 \mathrm{~S}$ & $120: 38 \cdot 76 \mathrm{E}$ \\
\hline 28. & HONSHUtr(03/25/86) & $04 \cdot 00-43 \cdot 3$ & 144 & $37: 15 \cdot 72 \mathrm{~N}$ & $139: 25 \cdot 38 E$ \\
\hline 29. & BRAZILza(03/26/86) & $22 \cdot 06 \cdot 57 \cdot 6$ & 609 & $07: 07 \cdot 50 \mathrm{~S}$ & $71: 38 \cdot 28 W$ \\
\hline 30. & NBRTANzo(04/03/86) & $07.39 \cdot 53.9$ & 30 & $06: 20 \cdot 40 \mathrm{~S}$ & $151: 42 \cdot 90 \mathrm{E}$ \\
\hline 31. & KURILEzc(04/05/86) & $22 \cdot 59 \cdot 09 \cdot 4$ & 80 & $44.27 .96 \mathrm{~N}$ & $147: 51 \cdot 42 \mathrm{E}$ \\
\hline 32. & MINDNOtr(04/09/86) & $21 \cdot 56 \cdot 19 \cdot 6$ & 56 & $09: 58 \cdot 14 \mathrm{~N}$ & $126: 09 \cdot 24 \mathrm{E}$ \\
\hline 33. & MOLUCAzc(04/10/86) & $02 \cdot 21 \cdot 13 \cdot 3$ & 33 & $00: 58 \cdot 14 S$ & $126: 50-28 \mathrm{E}$ \\
\hline 34. & MARANAzo(04/13/86) & $03 \cdot 00-20-9$ & 304 & $17: 11 \cdot 28 \mathrm{~N}$ & $145: 37 \cdot 26 \mathrm{E}$ \\
\hline 35. & VANATUzo(04/14/86) & $00 \cdot 25 \cdot 12 \cdot 4$ & 33 & $13: 56 \cdot 76 \mathrm{~S}$ & $166: 58 \cdot 62 \mathrm{E}$ \\
\hline 36. & KURILEzo(04/16/86) & $12 \cdot 52 \cdot 19 \cdot 7$ & 52 & $43: 52 \cdot 98 \mathrm{~N}$ & $147: 33.96 \mathrm{E}$ \\
\hline 37. & TANMBRza(04/18/86) & $08 \cdot 08 \cdot 39 \cdot 2$ & 44 & $06: 00-42 S$ & $131: 32-82 \mathrm{E}$ \\
\hline 38. & WIRIANzc(04/20/86) & $07-03 \cdot 30 \cdot 8$ & 33 & $02: 22 \cdot 50 \mathrm{~S}$ & $139: 19 \cdot 08 \mathrm{E}$ \\
\hline 39. & CRETE $\operatorname{tr}(04 / 27 / 86)$ & $09 \cdot 27 \cdot 02 \cdot 3$ & 10 & $34: 38 \cdot 46 \mathrm{~N}$ & $23: 24 \cdot 42 \mathrm{E}$ \\
\hline 40. & SUMTRAza(04/29/86) & $13 \cdot 59 \cdot 21 \cdot 8$ & 47 & $04: 24 \cdot 00 \mathrm{~N}$ & $95: 00 \cdot 24 \mathrm{E}$ \\
\hline 41. & RYUKYUtr(04/30/86) & $23 \cdot 14 \cdot 43 \cdot 7$ & 44 & $28: 42 \cdot 66 \mathrm{~N}$ & $130: 02 \cdot 82 \mathrm{E}$ \\
\hline 42. & PAPUAGzc(06/24/86) & $03 \cdot 11 \cdot 30 \cdot 9$ & 102 & $04: 26 \cdot 88 \mathrm{~S}$ & $143: 56 \cdot 58 \mathrm{E}$ \\
\hline 43. & FLOREStr(06/27/86) & $03 \cdot 09 \cdot 50 \cdot 8$ & 229 & $07: 56 \cdot 82 S$ & $122: 47 \cdot 46 \mathrm{E}$ \\
\hline 44. & FLORESpk(06/27/86) & $03 \cdot 09 \cdot 50 \cdot 8$ & 229 & $07: 56 \cdot 82 S$ & $122: 47 \cdot 46 \mathrm{E}$ \\
\hline 45. & TAGYKApk(06/29/86) & $21 \cdot 47 \cdot 59 \cdot 6$ & 20 & $05: 20-16 S$ & $20: 32 \cdot 34 \mathrm{E}$ \\
\hline 46. & MEXICOpk(07/05/86) & $22 \cdot 09 \cdot 36 \cdot 9$ & 112 & $15: 28 \cdot 02 \mathrm{~N}$ & $92: 34.92 \mathrm{~W}$ \\
\hline 47. & CARBRGIb(07/07/86) & $16 \cdot 26 \cdot 56 \cdot 6$ & 8 & $10: 23 \cdot 34 N$ & $56: 49 \cdot 92 \mathrm{E}$ \\
\hline 48. & MINAHAzc(07/08/86) & $04 \cdot 27 \cdot 34 \cdot 6$ & 245 & $01: 59 \cdot 82 \mathrm{~N}$ & $124: 18 \cdot 30 \mathrm{E}$ \\
\hline 49. & MOLUCApk(07/09/86) & $23 \cdot 10-53 \cdot 1$ & 28 & $01: 54 \cdot 24 \mathrm{~N}$ & $126: 31 \cdot 50 \mathrm{E}$ \\
\hline 50. & SIRANfb $(07 / 12 / 86)$ & $07 \cdot 54 \cdot 26 \cdot 8$ & 10 & $29: 57 \cdot 72 \mathrm{~N}$ & $51: 34.92 \mathrm{E}$ \\
\hline
\end{tabular}


Table 2. (continued)

\begin{tabular}{|c|c|c|c|c|c|}
\hline \multirow{2}{*}{$\begin{array}{l}\text { Sl. } \\
\text { No. } \\
51 .\end{array}$} & \multirow{2}{*}{$\begin{array}{c}\text { Source region } \\
\text { AFUSSRzc(07/17/86) }\end{array}$} & \multirow{2}{*}{$\frac{\text { Origin time }}{15 \cdot 46 \cdot 37 \cdot 0}$} & \multirow{2}{*}{$\begin{array}{c}\begin{array}{c}\text { Depth } \\
(\mathrm{km})\end{array} \\
47\end{array}$} & \multicolumn{2}{|c|}{ Coordinates } \\
\hline & & & & $36: 40-08 \mathrm{~N}$ & $71: 14 \cdot 82 \mathrm{E}$ \\
\hline 52. & FOXIS zq(07/19/86) & $04 \cdot 31 \cdot 55.9$ & 33 & $53: 21 \cdot 12 \mathrm{~N}$ & $165: 52.92 W$ \\
\hline 53. & KURILEzc(07/19/86) & $05 \cdot 59 \cdot 36 \cdot 2$ & 141 & $47: 15 \cdot 84 \mathrm{~N}$ & 151:07.62E \\
\hline 54. & BURMAtr(07/26/86) & $20 \cdot 24 \cdot 47 \cdot 8$ & 27 & $23: 45 \cdot 18 \mathrm{~N}$ & $94: 10-62 E$ \\
\hline 55. & EUSSRpk(07/26/86) & $14 \cdot 46 \cdot 18 \cdot 9$ & 310 & $45: 25 \cdot 32 \mathrm{~N}$ & $137: 04 \cdot 20 \mathrm{E}$ \\
\hline 56. & CHINApk(08/06/86) & $19 \cdot 55 \cdot 15 \cdot 6$ & 34 & $29: 20-64 N$ & $100: 54.90 \mathrm{~W}$ \\
\hline 57. & PHILPNzo(08/09/86) & $00-53 \cdot 12 \cdot 6$ & 80 & $14.05 .94 \mathrm{~N}$ & $120: 20-10 \mathrm{E}$ \\
\hline 58. & HALMRAzc(08/10/86) & $04 \cdot 40 \cdot 49 \cdot 7$ & 104 & $01: 59 \cdot 10 \mathrm{~N}$ & $128: 16 \cdot 26 \mathrm{E}$ \\
\hline 59. & MOLUCAtr(08/17/86) & $15 \cdot 27 \cdot 41 \cdot 2$ & 31 & $02: 16 \cdot 56 \mathrm{~N}$ & $126: 57 \cdot 54 \mathrm{E}$ \\
\hline 60. & BANDASzo(08/19/86) & $12 \cdot 52 \cdot 41 \cdot 1$ & 33 & $04: 07: 32 S$ & $129: 19: 44 E$ \\
\hline 61. & PHILPNzo(08/19/86) & $22 \cdot 41 \cdot 35 \cdot 8$ & 98 & $12: 30-84 \mathrm{~N}$ & $124: 28 \cdot 68 \mathrm{E}$ \\
\hline 62. & SIOCENtr(08/20/86) & $21 \cdot 15 \cdot 46 \cdot 4$ & 10 & $01: 58-38 \mathrm{~S}$ & $87.05 \cdot 28 E$ \\
\hline 63. & ROMANAzc(08/30/86) & $21 \cdot 28 \cdot 35 \cdot 4$ & 132 & $45: 32.82 \mathrm{~N}$ & $26: 18 \cdot 86 \mathrm{E}$ \\
\hline 64. & VOLCNOpk(08/31/86) & $09 \cdot 23 \cdot 04 \cdot 7$ & 46 & $23: 02 \cdot 52 \mathrm{~N}$ & 144:07.20E \\
\hline 65. & KURILEtr(09/01/86) & $21 \cdot 47 \cdot 35 \cdot 0$ & 148 & $46: 37 \cdot 86 \mathrm{~N}$ & $150: 00-60 \mathrm{E}$ \\
\hline 66. & HALMRAzc(09/09/86) & $15 \cdot 58 \cdot 22 \cdot 5$ & 33 & $00: 55 \cdot 62 \mathrm{~N}$ & $127: 56 \cdot 64 \mathrm{E}$ \\
\hline 67. & NBRTANtr(09/11/86) & $00 \cdot 18 \cdot 25 \cdot 5$ & 51 & $05: 11 \cdot 22 \mathrm{~S}$ & $152: 26 \cdot 52 \mathrm{E}$ \\
\hline 68. & MOLUCAtr(09/11/86) & $17 \cdot 54-03-0$ & 33 & $00: 28 \cdot 44 \mathrm{~N}$ & $125: 51 \cdot 72 \mathrm{E}$ \\
\hline 69. & HINDKUtr(09/13/86) & $14 \cdot 14 \cdot 54 \cdot 3$ & 200 & $36: 26.52 \mathrm{~N}$ & $70: 45 \cdot 96 \mathrm{E}$ \\
\hline 70. & SOLMONza(09/14/86) & $20 \cdot 58 \cdot 23 \cdot 1$ & 59 & $06: 28 \cdot 20 S$ & $154: 54 \cdot 96 \mathrm{E}$ \\
\hline 71. & ANDREOtr(09/15/86) & $06 \cdot 29 \cdot 35 \cdot 8$ & 33 & $51: 33 \cdot 66 \mathrm{~N}$ & $177.05 \cdot 10 \mathrm{~W}$ \\
\hline 72. & MARINAtr(09/16/86) & $18 \cdot 20 \cdot 17 \cdot 7$ & 48 & $19: 22 \cdot 56 \mathrm{~N}$ & $146: 18 \cdot 06 \mathrm{E}$ \\
\hline 73. & AFUSSRzd(09/17/86) & $12 \cdot 08 \cdot 09 \cdot 4$ & 120 & $37: 17 \cdot 40 \mathrm{~N}$ & $71: 43 \cdot 80 \mathrm{E}$ \\
\hline 74. & AFUSSRtr(09/17/86) & $12 \cdot 08-09 \cdot 4$ & 120 & $37: 17 \cdot 40 \mathrm{~N}$ & $71: 43 \cdot 80 \mathrm{E}$ \\
\hline 75. & CARBRGfb(09/17/86) & $21 \cdot 25 \cdot 15 \cdot 0$ & 10 & $10: 29 \cdot 82 \mathrm{~N}$ & $56: 59 \cdot 98 \mathrm{E}$ \\
\hline 76. & HONSHUzc(09/20/86) & $03 \cdot 04 \cdot 58 \cdot 7$ & 75 & $36: 27 \cdot 24 N$ & $140: 31 \cdot 20 \mathrm{E}$ \\
\hline 77. & BANDAza(09/22/86) & $16 \cdot 15 \cdot 05 \cdot 8$ & 123 & $06: 42 \cdot 96 \mathrm{~S}$ & $130: 25 \cdot 62 \mathrm{E}$ \\
\hline 78. & BONINza(09/27/86) & $19 \cdot 38 \cdot 42 \cdot 1$ & 30 & $27: 52-02 \mathrm{~N}$ & $142: 46-62 \mathrm{E}$ \\
\hline 79. & BANDASza(10/01/86) & $13 \cdot 00-06 \cdot 3$ & 343 & $05: 34-74 S$ & $128: 37-02 \mathrm{E}$ \\
\hline 80. & MEDRNSpk(10/02/86) & $10-12 \cdot 45 \cdot 6$ & 46 & $34: 50.76 \mathrm{~N}$ & $28: 18 \cdot 84 W$ \\
\hline 81. & HALMRAIr(10/04/86) & $02-00-08 \cdot 0$ & 106 & $02: 58.92 \mathrm{~N}$ & $128: 02 \cdot 16 \mathrm{E}$ \\
\hline 82. & ANDREOza $10 / 06 / 86)$ & $04 \cdot 21 \cdot 46 \cdot 7$ & 43 & $51: 51 \cdot 78 \mathrm{~N}$ & 176:15-42W \\
\hline 83. & SHONSUtr(10/07/86) & $11.40 .55 \cdot 0$ & 400 & $31: 54 \cdot 42 \mathrm{~N}$ & $137: 40 \cdot 56 \mathrm{E}$ \\
\hline 84. & JAVARzo(10/10/86) & $17 \cdot 48 \cdot 24 \cdot 6$ & 82 & $07: 29 \cdot 88 \mathrm{~S}$ & 107:13.98E \\
\hline 85. & HINDKUtr(10/13/86) & $16 \cdot 11 \cdot 40-4$ & 117 & $36: 04-02 \mathrm{~N}$ & $70: 50-88 \mathrm{E}$ \\
\hline 86. & HINDKUza(10/13/86) & $16 \cdot 11 \cdot 40 \cdot 4$ & 117 & $36: 04 \cdot 02 \mathrm{~N}$ & $70: 50-88 \mathrm{E}$ \\
\hline 87. & HONSHUtr(10/13/86) & $21 \cdot 17 \cdot 50-9$ & 65 & $37: 06-12 \mathrm{~N}$ & $141: 00-66 \mathrm{E}$ \\
\hline 88. & BANDASzo(10/17/86) & $07 \cdot 32 \cdot 51 \cdot 3$ & 67 & $05: 16 \cdot 32 S$ & $131: 25 \cdot 92 \mathrm{E}$ \\
\hline 89. & ANDREOpk(10/18/86) & $01 \cdot 02 \cdot 52 \cdot 1$ & 33 & $51: 43 \cdot 80 \mathrm{~N}$ & $175: 17 \cdot 10 \mathrm{~W}$ \\
\hline 90. & JAVAzo(10/18/86) & $22 \cdot 09 \cdot 31 \cdot 7$ & 643 & $05: 37 \cdot 86 \mathrm{~S}$ & $109: 59 \cdot 82 \mathrm{E}$ \\
\hline 91. & HALMRApk(10/21/86) & $21 \cdot 57 \cdot 59 \cdot 4$ & 142 & $01: 32 \cdot 46 \mathrm{~N}$ & $127: 25 \cdot 44 \mathrm{E}$ \\
\hline 92. & SACRUZtr(10/22/86) & $08 \cdot 59 \cdot 28 \cdot 8$ & 165 & $10.34 \cdot 14 S$ & $166: 02-40 \mathrm{E}$ \\
\hline 93. & EPGNEAzo(10/23/86) & $03 \cdot 54-20-8$ & 127 & $06: 05 \cdot 82 S$ & $146: 18 \cdot 36 E$ \\
\hline 94. & NCHILEpk(10/24/86) & $02 \cdot 42 \cdot 51 \cdot 6$ & 51 & $25: 19 \cdot 14 S$ & $70: 10.56 \mathrm{~W}$ \\
\hline 95. & NCHILEzd $10 / 24 / 86)$ & $02 \cdot 42 \cdot 51 \cdot 6$ & 51 & $25: 19 \cdot 14 S$ & $70: 10-56 \mathrm{~W}$ \\
\hline 96. & AIRISEpk(10/28/86) & $15 \cdot 11 \cdot 23 \cdot 3$ & 10 & $30: 28 \cdot 26 S$ & $60: 10-92 \mathrm{E}$ \\
\hline 97. & MINDNOtb(10/29/86) & $20-11 \cdot 39 \cdot 7$ & 72 & $05: 43 \cdot 32 \mathrm{~N}$ & $125: 19-86 \mathrm{E}$ \\
\hline
\end{tabular}


Table 2. (continued)

\begin{tabular}{|c|c|c|c|c|c|}
\hline \multirow{2}{*}{$\begin{array}{l}\text { S!. } \\
\text { No. } \\
98 .\end{array}$} & \multirow{2}{*}{$\frac{\text { Source region }}{\text { MINDNOzo(10/29/86) }}$} & \multirow{2}{*}{ Origin time } & \multirow{2}{*}{$\begin{array}{c}\begin{array}{c}\text { Depth } \\
(\mathrm{km})\end{array} \\
72\end{array}$} & \multicolumn{2}{|c|}{ Coordinates } \\
\hline & & & & $05: 43 \cdot 32 \mathrm{~N}$ & $125: 19 \cdot 86 \mathrm{E}$ \\
\hline 99. & BURMAzo(11/01/86) & $05 \cdot 02 \cdot 42 \cdot 4$ & 26 & $26: 54 \cdot 12 \mathrm{~N}$ & $96: 25 \cdot 50 \mathrm{E}$ \\
\hline 100. & MINDNOzo(11/06/86) & $02 \cdot 48 \cdot 23 \cdot 6$ & 62 & $09: 00 \cdot 42 \mathrm{~N}$ & $126: 14 \cdot 40 \mathrm{E}$ \\
\hline 101. & ANDREOzo(11/06/86) & $18 \cdot 27 \cdot 001$ & 33 & $51: 28 \cdot 14 \mathrm{~N}$ & $176: 41.04 \mathrm{~W}$ \\
\hline 102. & MOLUCAfb(11/11/86) & $00 \cdot 02 \cdot 32 \cdot 0$ & 79 & $02: 24 \cdot 84 \mathrm{~N}$ & $126: 48 \cdot 66 \mathrm{E}$ \\
\hline 103. & MOLUCAzo(11/11/86) & $00-02 \cdot 32 \cdot 0$ & 79 & $02: 24 \cdot 84 \mathrm{~N}$ & $126: 48 \cdot 66 \mathrm{E}$ \\
\hline 104. & TAKXJGIb(11/12/86) & $10-06 \cdot 15 \cdot 3$ & 115 & $38: 27 \cdot 30 \mathrm{~N}$ & $73: 16 \cdot 68 \mathrm{E}$ \\
\hline 105. & TAKXJG20 $(11 / 12 / 86)$ & $10-06 \cdot 15 \cdot 3$ & 115 & $38: 27 \cdot 30 \mathrm{~N}$ & $73: 16-68 E$ \\
\hline 106. & TAIWANtr(11/14/86) & $21 \cdot 20 \cdot 10 \cdot 5$ & 34 & $23: 54 \cdot 06 \mathrm{~N}$ & $121: 34 \cdot 44 E$ \\
\hline 107. & TAIWANzo(11/14/86) & $23 \cdot 04 \cdot 37 \cdot 0$ & 33 & $23.51 .96 \mathrm{~N}$ & $121: 42 \cdot 66 \mathrm{E}$ \\
\hline 108. & PRUECQfb(11/23/86) & $01 \cdot 39 \cdot 23 \cdot 9$ & 106 & $03: 20-52 S$ & $77: 24-66 \mathrm{~W}$ \\
\hline 109. & PRUECQza(11/23/86) & $01 \cdot 39 \cdot 23 \cdot 9$ & 106 & $03: 20-52 S$ & $77: 24 \cdot 66 \mathrm{~W}$ \\
\hline 110. & HJAPANzo(11/28/86) & $22 \cdot 29 \cdot 35 \cdot 1$ & 41 & $36: 20 \cdot 70 \mathrm{~N}$ & $141: 10-44 \mathrm{E}$ \\
\hline 111. & HPAJANpk(11/30/86) & $20 \cdot 15 \cdot 30-3$ & 37 & $38: 51 \cdot 18 \mathrm{~N}$ & $141: 56 \cdot 88 \mathrm{E}$ \\
\hline 112. & NIBRISpk(12/07/86) & $05 \cdot 40 \cdot 39 \cdot 2$ & 215 & $06: 46.98 \mathrm{~N}$ & $95: 07 \cdot 20 \mathrm{E}$ \\
\hline 113. & SOLMISfb(12/11/86) & $19-56 \cdot 12 \cdot 5$ & 61 & $10: 29 \cdot 28 S$ & $160: 42 \cdot 90 \mathrm{E}$ \\
\hline 114. & HJAPANIb $(12 / 14 / 86)$ & $00-11 \cdot 31 \cdot 2$ & 352 & $32: 43 \cdot 62 \mathrm{~N}$ & $137: 43 \cdot 38 E$ \\
\hline 115. & AFUSSRzo(12/17/86) & $08 \cdot 31 \cdot 30-3$ & 225 & $36: 32 \cdot 34 \mathrm{~N}$ & $71: 07 \cdot 50 \mathrm{E}$ \\
\hline 116. & AFUSSRzo(12/18/86) & $07 \cdot 23 \cdot 30-0$ & 182 & $36: 34.08 \mathrm{~N}$ & $71: 21 \cdot 18 \mathrm{E}$ \\
\hline 117. & ANDREOM(12/19/86) & $13 \cdot 50-10 \cdot 3$ & 33 & $51: 31.02 \mathrm{~N}$ & $176: 58.62 \mathrm{~W}$ \\
\hline 118. & ANDREOtr(12/19/86) & $13 \cdot 50 \cdot 10 \cdot 3$ & 33 & $51: 31.02 \mathrm{~N}$ & $176: 58.62 W$ \\
\hline 119. & TAIWANzo(12/21/86) & $02 \cdot 42 \cdot 49 \cdot 0$ & 275 & $25: 31.02 \mathrm{~N}$ & $121: 30-42 \mathrm{E}$ \\
\hline 120. & MARINAtr(01/01/87) & $16 \cdot 25 \cdot 34 \cdot 1$ & 74 & $02: 42 \cdot 90 \mathrm{~S}$ & $138: 21 \cdot 78 \mathrm{E}$ \\
\hline 121. & MARINAzo(01/01/87) & $16 \cdot 25 \cdot 34 \cdot 1$ & 74 & $02: 42.90 \mathrm{~S}$ & $138: 21 \cdot 78 \mathrm{E}$ \\
\hline 122. & CHINAZC(01/05/87) & $22 \cdot 52 \cdot 46 \cdot 5$ & 17 & $41: 57.84 \mathrm{~N}$ & $81: 19 \cdot 14 \mathrm{E}$ \\
\hline 123. & TAIWANfb(01/06/87) & $05 \cdot 07 \cdot 48 \cdot 1$ & 38 & $23: 58 \cdot 56 \mathrm{~N}$ & $121: 43 \cdot 74 \mathrm{E}$ \\
\hline 124. & TAIWANzo(01/06/87) & $05 \cdot 07 \cdot 48 \cdot 1$ & 38 & $23: 58 \cdot 56 \mathrm{~N}$ & $121: 43 \cdot 74 \mathrm{E}$ \\
\hline 125. & NIRELRfb(01/08/87) & $19 \cdot 48 \cdot 55 \cdot 4$ & 44 & $04: 44 \cdot 16 S$ & $153: 06 \cdot 30 \mathrm{E}$ \\
\hline 126. & NIRELRzo(01/08/87) & $19 \cdot 48 \cdot 55 \cdot 4$ & 44 & $04: 44 \cdot 16 \mathrm{~S}$ & $153: 06 \cdot 30 \mathrm{E}$ \\
\hline 127. & HONSHUza(01/09/87) & $06 \cdot 14 \cdot 44 \cdot 8$ & 68 & $39: 53 \cdot 70 \mathrm{~N}$ & $141: 40-62 \mathrm{E}$ \\
\hline 128. & HOKKAIzo(01/14/87) & 11.03 .48 .7 & 102 & $42: 33 \cdot 90 \mathrm{~N}$ & $142: 51 \cdot 00 \mathrm{E}$ \\
\hline 129. & KAMCHTzo(01/19/87) & 06.47 .43 .0 & 42 & $54: 44 \cdot 64 \mathrm{~N}$ & $163: 16 \cdot 62 E$ \\
\hline 130. & NEPALzo(01/19/87) & $07.46 \cdot 24 \cdot 4$ & 33 & $28: 23 \cdot 10 \mathrm{~N}$ & $83: 40-92 \mathrm{E}$ \\
\hline 131. & NEPALzq(01/19/87) & $08 \cdot 12 \cdot 05 \cdot 8$ & 33 & $28: 14 \cdot 58 \mathrm{~N}$ & $83: 34 \cdot 32 \mathrm{E}$ \\
\hline 132. & MARINAzo(01/21/87) & $11 \cdot 26 \cdot 36 \cdot 6$ & 118 & $20: 36 \cdot 48 \mathrm{~N}$ & $144: 53 \cdot 10 \mathrm{E}$ \\
\hline 133. & MOLUCAIb(01/23/87) & $17 \cdot 51.09 \cdot 2$ & 72 & $01: 38.76 \mathrm{~N}$ & $126: 31.86 \mathrm{E}$ \\
\hline 134. & MOLUCAzd(01/23/87) & $17 \cdot 51 \cdot 09 \cdot 2$ & 72 & $01: 38.76 \mathrm{~N}$ & $126: 31 \cdot 86 \mathrm{E}$ \\
\hline 135. & HINDKUtr(05/05/87) & $15 \cdot 40-47.5$ & 208 & $36: 28 \cdot 80 \mathrm{~N}$ & $70: 40-38 \mathrm{E}$ \\
\hline 136. & KAZAK Hzo(05/06/87) & $04.02 .05 \cdot 6$ & 1 & $49: 49 \cdot 80 \mathrm{~N}$ & $78: 07 \cdot 50 \mathrm{E}$ \\
\hline 137. & ANDREOzc(05/06/87) & $04 \cdot 06 \cdot 14 \cdot 1$ & 20 & $51: 16 \cdot 32 \mathrm{~N}$ & $179: 53.88 \mathrm{~W}$ \\
\hline 138. & NBRITNzo(05/06/87) & $12 \cdot 39 \cdot 49 \cdot 1$ & 20 & $05: 42.90 \mathrm{~S}$ & $152: 39 \cdot 36 \mathrm{E}$ \\
\hline 139. & EUSSRza(05/07/87) & $03.05 \cdot 49 \cdot 1$ & 430 & $46: 44 \cdot 16 \mathrm{~N}$ & $139: 13.92 E$ \\
\hline 140. & BALIStr(05/10/87) & $00-37 \cdot 100$ & 42 & $07: 44 \cdot 22 S$ & $115: 59 \cdot 82 \mathrm{E}$ \\
\hline 141. & TALAUDzc(05/11/87) & $09 \cdot 59 \cdot 34 \cdot 1$ & 94 & $04: 28 \cdot 20 \mathrm{~N}$ & $127: 42 \cdot 54 \mathrm{E}$ \\
\hline 142. & MINDNOzc(05/12/87) & $01 \cdot 30 \cdot 25 \cdot 0$ & 25 & $07.05 \cdot 40 \mathrm{~N}$ & $126: 42 \cdot 06 \mathrm{E}$ \\
\hline 143. & ICELNDzc(05/25/87) & $11 \cdot 31 \cdot 54 \cdot 3$ & 8 & $63: 51 \cdot 00 \mathrm{~N}$ & $19: 43.68 W$ \\
\hline 144. & BANDASzc(05/30/87) & $16 \cdot 54 \cdot 04 \cdot 7$ & 138 & $06: 03 \cdot 84 \mathrm{~S}$ & $130: 31 \cdot 08 \mathrm{E}$ \\
\hline
\end{tabular}


Table 2. (continued)

\begin{tabular}{|c|c|c|c|c|c|}
\hline \multirow{2}{*}{$\begin{array}{l}\text { Sl. } \\
\text { No. } \\
145 .\end{array}$} & \multirow{2}{*}{$\frac{\text { Source region }}{\text { KURILEzc(05/30/87) }}$} & \multirow{2}{*}{$\frac{\text { Origin time }}{17 \cdot 19 \cdot 00 \cdot 1}$} & \multirow{2}{*}{$\frac{\begin{array}{c}\text { Depth } \\
(\mathrm{km})\end{array}}{53}$} & \multicolumn{2}{|c|}{ Coordinates } \\
\hline & & & & $44: 40-14 \mathrm{~N}$ & $150: 17 \cdot 34 \mathrm{E}$ \\
\hline 146. & MINHSAzc(05/31/87) & $18 \cdot 32 \cdot 17 \cdot 0$ & 79 & $00: 44 \cdot 88 \mathrm{~N}$ & $121: 56.46 \mathrm{E}$ \\
\hline 147. & ANDREOzc(06/01/87) & $00 \cdot 15 \cdot 14 \cdot 3$ & 33 & $51: 32 \cdot 40 \mathrm{~N}$ & $177: 30 \cdot 54 W$ \\
\hline 148. & CHINAzc(06/05/87) & $04 \cdot 59 \cdot 58 \cdot 3$ & 1 & $41: 35.04 \mathrm{~N}$ & $88: 44 \cdot 22 \mathrm{E}$ \\
\hline 149. & PHILIPtr(06/05/87) & $21 \cdot 25 \cdot 11 \cdot 2$ & 45 & $05: 22 \cdot 86 \mathrm{~N}$ & $127: 32 \cdot 04 \mathrm{E}$ \\
\hline 150. & PHILIPtr(06/05/87) & $22 \cdot 00-03 \cdot 1$ & 47 & $05: 15.90 \mathrm{~N}$ & $127: 30.78 E$ \\
\hline 151. & PHILIPzc(06/07/87) & $05 \cdot 49 \cdot 43 \cdot 6$ & 15 & $20: 25 \cdot 74 \mathrm{~N}$ & $121: 21 \cdot 96 \mathrm{E}$ \\
\hline 152. & XURILESB(06/13/87) & $14 \cdot 00 \cdot 39 \cdot 3$ & 42 & $44: 40-26 \mathrm{~N}$ & $150.23 \cdot 52 \mathrm{E}$ \\
\hline 153. & TALAUDtr(06/15/87) & $06 \cdot 31 \cdot 44.7$ & 136 & $03: 54 \cdot 94 \mathrm{~N}$ & $125: 56.94 \mathrm{E}$ \\
\hline 154. & KAZAKHzc(06/20/87) & $00.53 \cdot 04 \cdot 8$ & 1 & $49: 54 \cdot 78 \mathrm{~N}$ & $78: 4410 \mathrm{E}$ \\
\hline 155. & IRIANtr(06/27/87) & $00 \cdot 17 \cdot 04 \cdot 6$ & 21 & $02: 09 \cdot 84 \mathrm{~S}$ & $138: 10 \cdot 20 \mathrm{E}$ \\
\hline 156. & KYUSHUzc(07/03/87) & $10-10-43 \cdot 7$ & 168 & $31: 11 \cdot 76 \mathrm{~N}$ & $130: 19 \cdot 32 \mathrm{E}$ \\
\hline 157. & CHAGOStr(07/03/87) & $18 \cdot 03 \cdot 59 \cdot 7$ & 26 & $06: 47 \cdot 16 \mathrm{~S}$ & $72: 14 \cdot 82 E$ \\
\hline 158. & ALEUTNpk(07/05/87) & $09 \cdot 23 \cdot 00 \cdot 0$ & 33 & $51: 29 \cdot 16 \mathrm{~N}$ & $174: 39 \cdot 60 \mathrm{E}$ \\
\hline 159. & ALEUTNpk(07/06/87) & $00-03 \cdot 25 \cdot 6$ & 33 & $51: 30 \cdot 48 \mathrm{~N}$ & $174: 43 \cdot 26 \mathrm{E}$ \\
\hline 160. & TALAUDpk(07/07/87) & $10 \cdot 36 \cdot 03 \cdot 7$ & 70 & $05: 24 \cdot 18 \mathrm{~N}$ & $125: 01 \cdot 68 \mathrm{E}$ \\
\hline 161. & KURILEzc(07/08/87) & $22 \cdot 56-02 \cdot 7$ & 152 & $46: 26 \cdot 22 \mathrm{~N}$ & $149: 33-48 E$ \\
\hline 162. & TALAUDzc(07/14/87) & $04 \cdot 31 \cdot 23 \cdot 0$ & 33 & $03: 50.04 \mathrm{~N}$ & $126: 32 \cdot 52 \mathrm{E}$ \\
\hline 163. & OKHTSKzc(07/14/87) & $23 \cdot 46 \cdot 03 \cdot 5$ & 591 & $49: 37 \cdot 86 \mathrm{~N}$ & $147: 49 \cdot 68 \mathrm{E}$ \\
\hline 164. & HONSHUtr(07/16/87) & $05 \cdot 46 \cdot 29 \cdot 5$ & 310 & $33: 03 \cdot 54 \mathrm{~N}$ & $138: 05 \cdot 76 \mathrm{E}$ \\
\hline 165. & KAZAKHpk\{07/17/87\} & 01.1707 .0 & 1 & $49: 4794 \mathrm{~N}$ & $78.06-60 \mathrm{E}$ \\
\hline 166. & TALAUDtr(07/22/87) & $08 \cdot 03 \cdot 17 \cdot 2$ & 51 & $04: 03.06 \mathrm{~N}$ & $125: 34 \cdot 92 \mathrm{E}$ \\
\hline 167. & MINHASFb(07/31/87) & $00 \cdot 27 \cdot 32 \cdot 0$ & 167 & $00: 10.50 \mathrm{~N}$ & $123: 36 \cdot 30 E$ \\
\hline 168. & KAZAKHzc(08/02/87) & $00.58 \cdot 06 \cdot 8$ & 1 & $49: 52 \cdot 80 \mathrm{~N}$ & $78: 55.02 E$ \\
\hline
\end{tabular}

Note: Code after event name indicates pick: $\mathrm{b}$, first break; $z c$, zero crossing; pk peak; tr, trough. There are a few multiple readings in the data set.

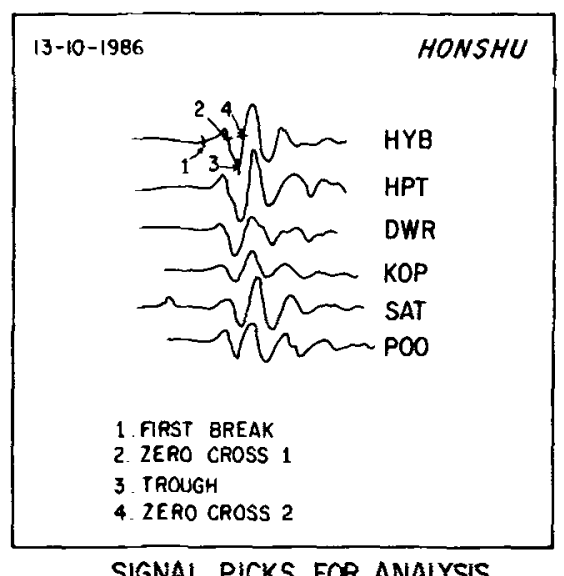

Figure 2. Seismograms showing how arrival-time are made. 


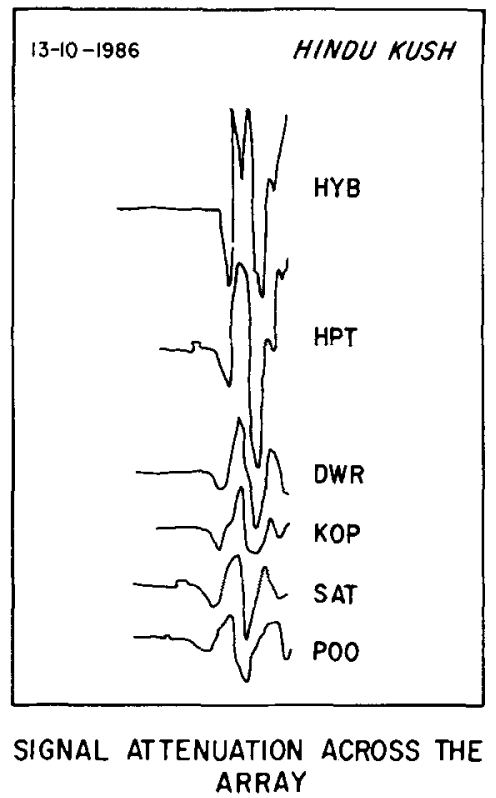

Figure 3. Signal attenuation and signal-shape changes observed at some of the stations.

station because of its location on Precambrian granite outside the DVP. A comparison of residuals at HYB with those at Srisailam-a station also on Precambrian granite about $150 \mathrm{~km}$ south-southeast of HYB - supports our assumptions that stations on the Precambrian granite are "normal" compared to those over the Deccan basalts.

For each station, the data were grouped into four categories according to epicentral distances: all distances, closer than $30^{\circ}, 30^{\circ}$ to $70^{\circ}$ and beyond $70^{\circ}$. We first examine the data from Poona (POO) where the largest data set is available (figure 4). Note that the range of variation of residuals is about $2 \mathrm{~s}$ varying from $1 \mathrm{~s}$ in the north to $-1 \mathrm{~s}$ in the east (figure $4 \mathrm{a}$ ). The relative residuals also depend on the epicentral distance and which determines the angle of emergence at the seismic station. Observe that for distances $<30^{\circ}$ the relative residuals are mostly positive (about $+1 \mathrm{~s}$ ) for all azimuths (figure $4 \mathrm{~b}$ ), with strong azimuthal variation occurring in the $30^{\circ}$ to $70^{\circ}$ range (figure $4 \mathrm{c}$ ) and a less pronounced but still clear variation occurring for distances $>70^{\circ}$ (figure 4d). Comparison of the relative residuals along the east-southeasterly profile from BHA, the westernmost station, to GUL on the eastern margin of DVP shows the pattern of residuals at BHA to be quite similar to that at POO but with larger variations from $+1.5 \mathrm{~s}$ to $-1.8 \mathrm{~s}$ (figure 5). Relative residuals at UJN, SOP and GUL show a similar azimuthal variation, but with a progressive decrease in magnitude towards HYB (figure 5). GUL, closest to HYB, shows the smallest residuals and is a key station to substantiate our argument that the strong azimuthal variation observed at other stations are associated with some anomaly in the deep structure beneath the DVP. The relative residuals along the southeasterly profile from DHL to AUB, BIR and LTR (figure 6) also show a decreasing azimuthal variation towards the eastern margin of the DVP, though LTR has five strong negative residuals in the $40-100^{\circ}$ azimuth range and $30-70^{\circ}$ distance range, cautioning against simplified inferences. Note that the residuals at these stations for distances $<30^{\circ}$ are generally negative, 

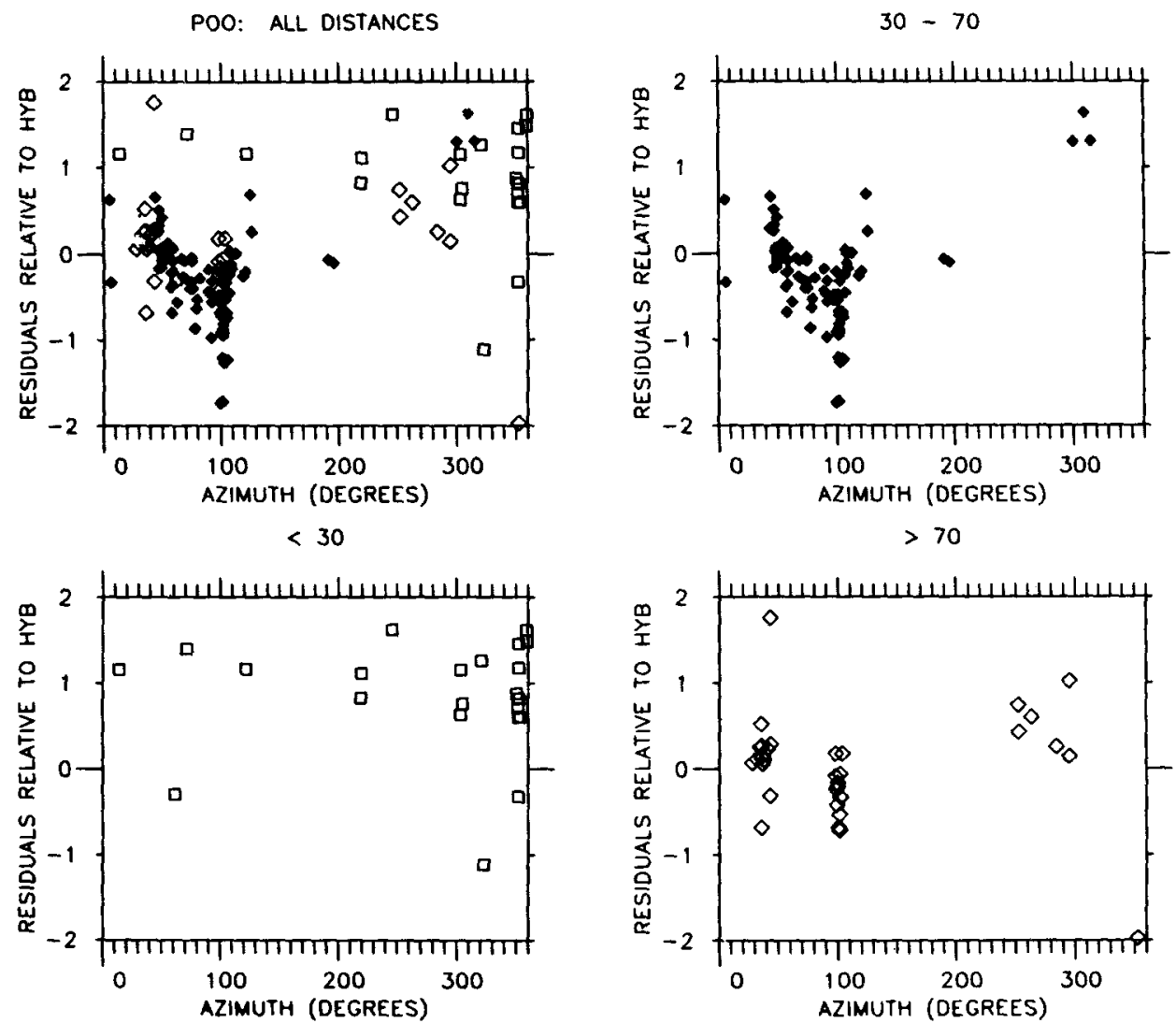

Figure 4. Relative residuals at POO with respect to HYB plotted as a function of azimuth of arrival. (a) All distances; hollow squares represent events at distances $<30^{\circ}$; solid diamonds represent events in the distance range of $30-70^{\circ}$; hollow diamonds represent events at distances $>70^{\circ}$. (b) Events at distances $<30^{\circ}$. (c) Events in the distance range $30-70^{\circ}$. (d) Events at distances $>70^{\circ}$.

unlike those observed at other stations. The southerly profile, comprised of stations SAT, KOP and DWR (figure 7) shows a behaviour similar to BHA and POO (figures 4 and 5). One surprise is at the southernmost station HPT (figure 7), south of GUL (figure 5) but outside the DVP. This station behaves similar to the western stations implying that the velocity anomaly responsible for the observed azimuthal variations extends even outside the DVP to the southeast.

Note the abrupt transition of residuals from negative values to positive near the $100^{\circ}$ azimuth range observed at all stations in the experimental area. In order to test whether this feature in the azimuthal pattern of residuals could be due to a positive residual bias at $\mathrm{HYB}$, we examined the relative residual patterns using the average event residual as reference and found that no such bias exists. The relative residuals thus calculated retained this feature at all these stations including Hyderabad, implying that it could not have arisen from any fortuitous cause related to the choice of Hyderabad as a reference station. We feel that this sharp transition in the residual patterns reflects a sharp velocity transition in the upper mantle outside the region being modelled. 


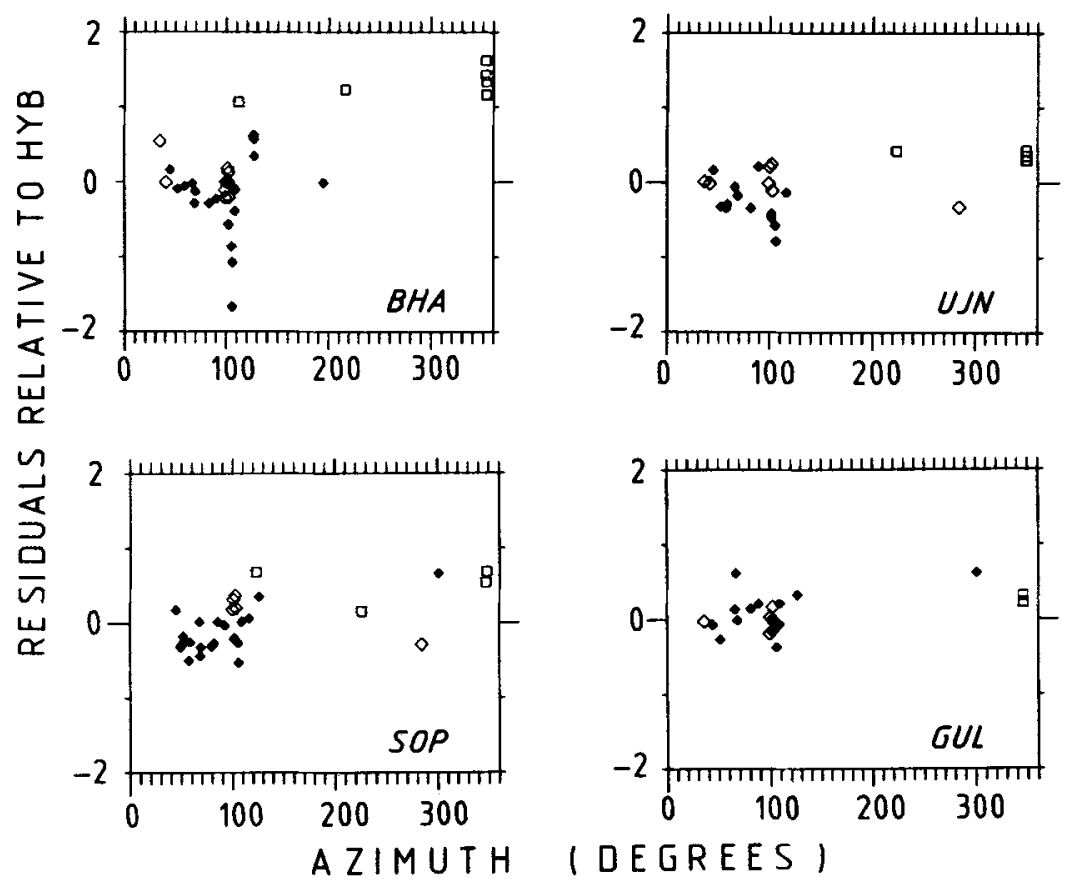

Figure 5. Relative residuals at BHA, UJN, SOP and GUL.
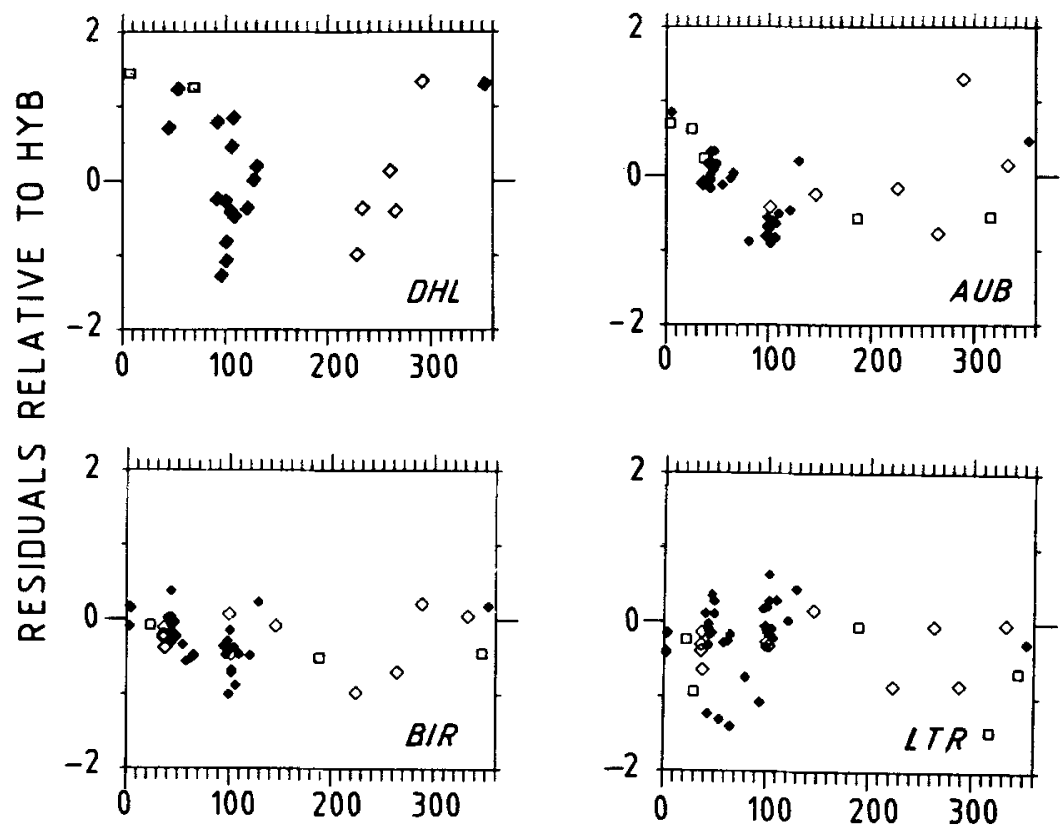

A ZIMUTH (DEGREES)

Figure 6. Relative residuals at DHL, BIR and LTR. 

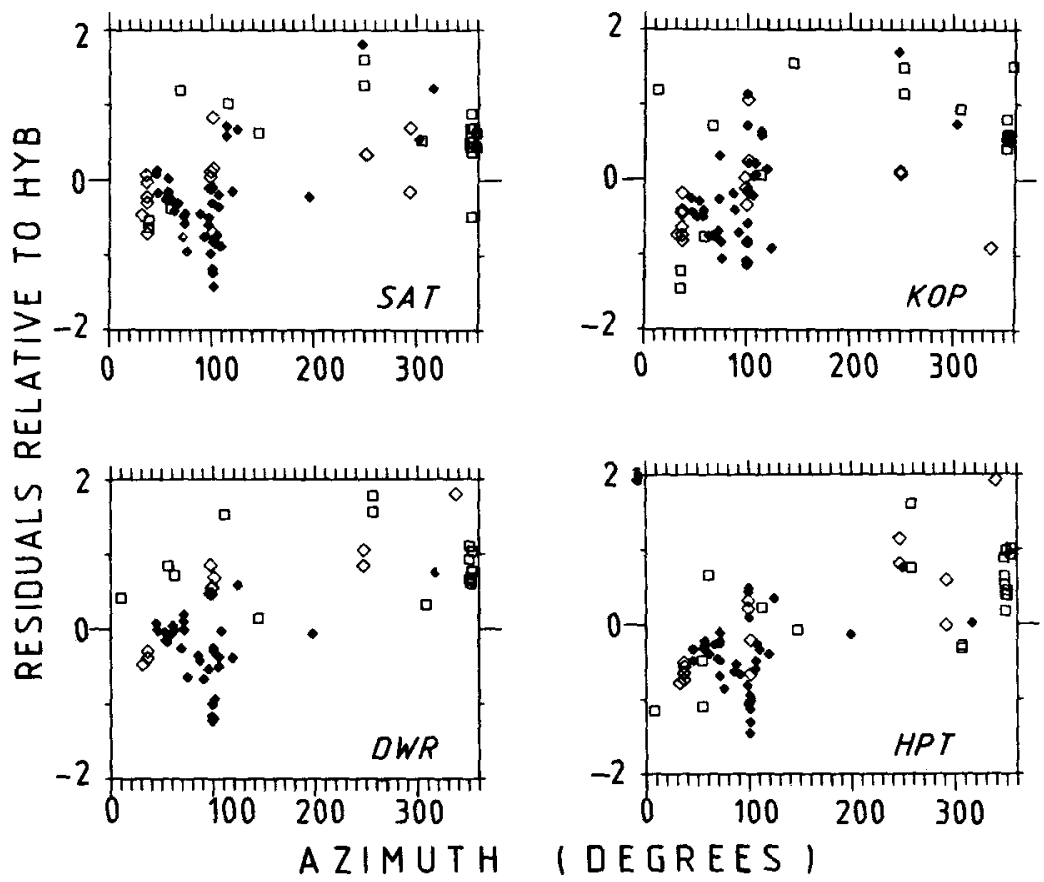

Figure 7. Relative residuals at SAT, KOP, DWR and HPT.

\section{Analysis using the simple ray plotting technique}

In this section we use simple ray-plotting techniques to show that the azimuthal and spatial variation of the relative residuals discussed above can be explained only in terms of velocity perturbations in the upper mantle of the DVP region. But first we evaluate the possible contributions that surficial and crustal layers can make to the residuals.

Variation in the thickness of basalt cover which attains a maximum of about $2 \mathrm{~km}$ in the western part, can given rise to a maximum relative residual of $+0.04 \mathrm{~s}$ assuming the $P$-wave velocity of the Deccan basalts and the basement rock to be $4.8 \mathrm{~km} / \mathrm{s}$ and $6.0 \mathrm{~km} / \mathrm{s}$ respectively (Kaila 1981; Kaila 1982). Further, even a $1.0 \mathrm{~km}$ thick low-velocity sedimentary layer with a velocity of $4.0 \mathrm{~km} / \mathrm{s}$ underlying the Deccan Traps, such as that found by Kaila et al (1981) in the Saurashtra Peninsula, will contribute a residual of less than $0.1 \mathrm{~s}$. This is even lower than the noise level in our experiment.

\subsection{Effect of the variation of crustal thickness}

The sub-trappean crustal model in the Koyna area of Deccan basalts has been approximated by three layers of 10,15 and $15 \mathrm{~km}$ thickness with average velocities of $5.7,6.5$ and $7.0 \mathrm{~km} / \mathrm{s}$ respectively (Kaila et al 1982). Variation in the thickness of any of these layers by as much as $5 \mathrm{~km}$ will give rise to a total residual of only about $0.1 \mathrm{~s}$. Crustal models of the Koyna region show that its thickness varies from $31.5 \mathrm{~km}$ near the coast to $39 \mathrm{~km}$ near Koyna (Kaila 1982). Even if we take the velocity contrast 
across the Moho to be bout $1.0 \mathrm{~km} / \mathrm{s}$ this order of variation in crustal thickness will only cause the arrivals to be $0.2 \mathrm{~s}$ earlier at the western stations compared with those at the eastern stations. The effect is approximately the same if the thinning of the crust is distributed over all the three layers.

It is thus clear that possible variations in the thicknesses of basalts, and sub-trappean sedimentary layers, if present, as well as those of the upper or of the whole crust can at the most account for only $+0 \cdot 2 \mathrm{~s}$ of the observed spatial variation of the relative residuals. The azimuthal variation cannot be accounted for by such shallow features at all. We therefore conclude that most of the observed residual pattern can only be accounted for by deep upper mantle features.

\subsection{Ray plotting onalysis}

To delineate qualitatively the zones of anomalous velocity structure responsible for the large azimuthally varying observed residuals, we first used a simple ray-tracing procedure similar to that used by Iyer et al (1981). From a knowledge of the emergent angles of seismic rays at various stations, inferred from the depth and epicentral distances of the respective teleseisms, rays are projected backwards from the various stations for each teleseism. The corresponding relative residuals are then projected on flat layers at three different depths $(100,200$ and $300 \mathrm{~km})$. If the observed residuals are due to the presence of an anomalous body rather than a random velocity perturbation, the whole set of projected residuals would be expected to show coherent signs and magnitudes so that they can be contoured when the depth chosen for the projection is correct. After considerable experimentation using data corresponding to $\Delta>30^{\circ}$, we found that the maximum coherence was achieved for projection at a depth of $200 \mathrm{~km}$. Also, some form of subjective averaging was needed to reduce too many overlapping numbers. Figure 8 shows the result of this projection to $200 \mathrm{~km}$ depth using the above constraints. The main inference from this figure is that it outlines, albeit broadly, a $200-\mathrm{km}$ wide north-south trending region of negative residuals of magnitude $>0.5 \mathrm{~s}$ extending from AUB in the north and probably continuing south beyond HPT. Since better constraints on the region of anomalous high velocity is provided by inversion of residuals which is presented below, we do not want to overinterpret this rather crude modelling of our data. However, we would like to state that this analysis, though not providing adequate constraints to delineate the anomalous structure beneath the Deccan Traps, suggests that the observed residuals are best interpreted in terms of a heterogeneous structure in the upper mantle consisting of higher-than-normal velocities beneath the south-central part of the DVP and lower-than-normal velocities in the west-central part.

\section{Tomographic results}

Ideally, to obtain well-constrained tomographic models, we need a set of teleseismic arrivals from events that are fairly well-distributed in various azimuths and distance ranges. Further, the arrival-time readings should be of high quality with minimal scatter, and the data set should be homogeneous and simultaneous at all stations in the seismic network. Though our experiment does not satisfy any of these criteria strictly, the similarity of inverted models for varying block and layer configurations, 


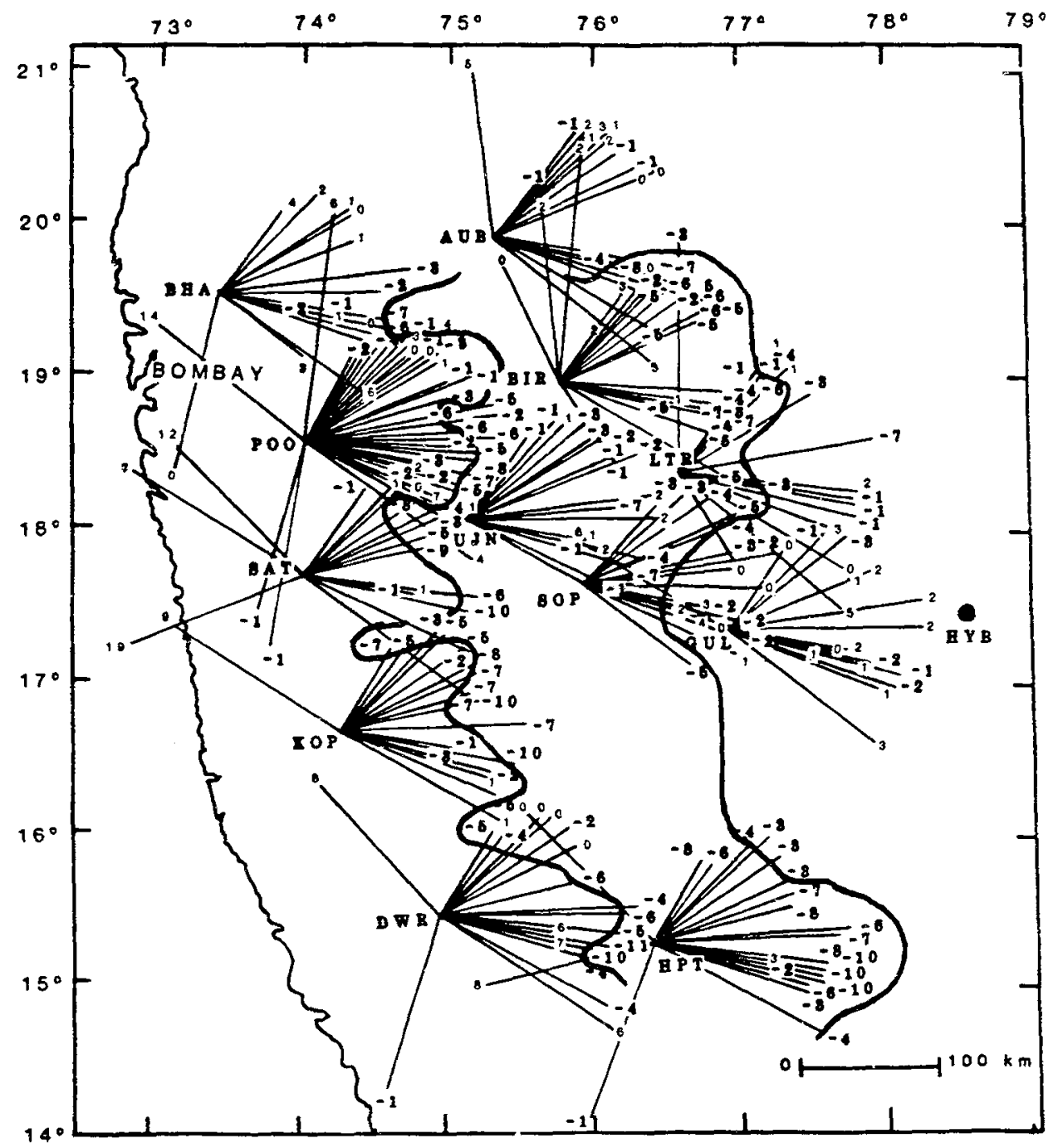

Figure 8. Selected averaged relative residuals projected at $200-\mathrm{km}$ depth.

suggests that they correctly resolve first-order features of the anomalous threedimensional P-velocity structure beneath the DVP. Also, these models agree with the qualitative inference derived from the azimuthal patterns of residuals using the ray-plotting approach discussed above. We performed numerous $\mathrm{ACH}$ inversions for a variety of layered models. The dimensions of the block were $200 \mathrm{~km}$ in the east-west direction and $150 \mathrm{~km}$ in the north-south direction. We use mantle velocities from Herrin's (1968b) earth model and the crustal velocity of $6.45 \mathrm{~km} / \mathrm{s}$ (Kaila 1982). For each inversion the respective resolution matrix and standard crrors were studied with a view to assessing the figure of merit of the solution. Note that $\mathrm{ACH}$ inversions use relative residuals based on the average of all available readings over the network for each event. Hence no specific reference stations were used. 


\section{Models}

Although a large number of models were used for inversion, description of a few of these models together with the corresponding data variance improvements are given in table 3. The main feature of the models, that is an anomalous high-velocity zone, appears to be significant only at sub-crustal depths. In order to constrain the upper boundary of the anomalous zone, we first analyse a series of two-layer models using all available data. These are shown in figure 9 . The numbers indicate velocity perturbations, positive numbers denoting higher-than-normal velocities and negative numbers

Table 3. Description of models.

\begin{tabular}{llc}
\hline No. of Layers & $\begin{array}{c}\text { Layer thicknesses } \\
(\mathrm{km})\end{array}$ & $\begin{array}{c}\text { Variance improvement } \\
(\%)\end{array}$ \\
\hline 2 & 40,60 & 16.0 \\
2 & 40,160 & 27.0 \\
2 & 40260 & $30-0$ \\
2 & 40,360 & 31.0 \\
3 & $100,300,200$ & $53-0$ \\
3 & $150,250,200$ & $63-0$ \\
4 & $40,160,200,200$ & 46.0 \\
\hline
\end{tabular}

MODEL $1: 40-100 \mathrm{Km}$

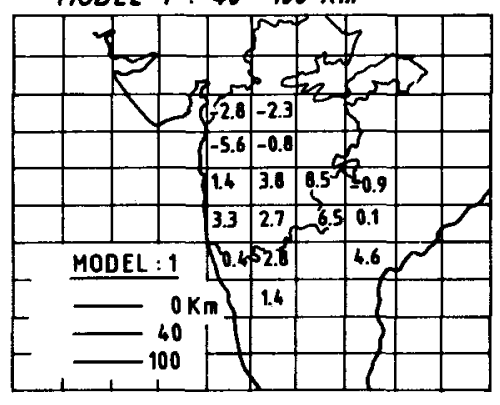

MODEL $2: 40-200 \mathrm{~km}$

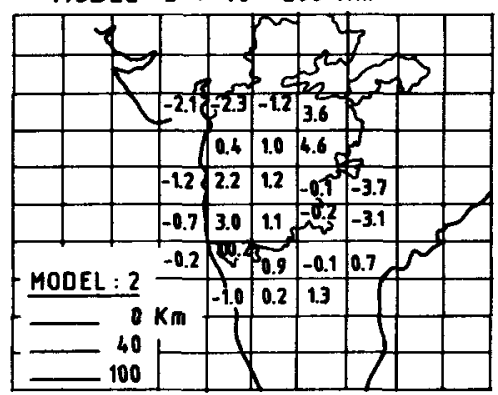

\section{$1400 \mathrm{Km}$}

MODEL $3: 40-300 \mathrm{~km}$

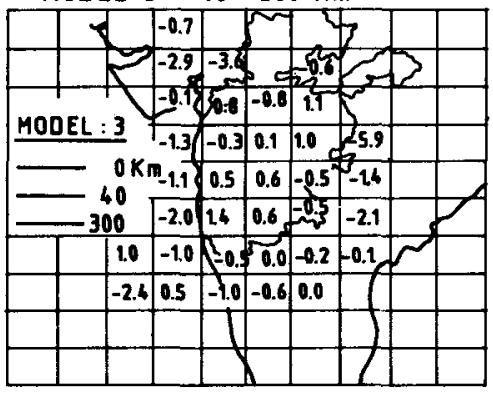

MODEL 4: $40-400 \mathrm{~km}$

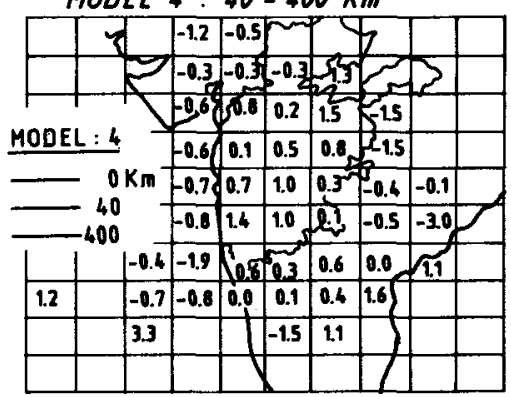

Figure 9. Second layer of 2-layer tomographic models derived using data at all available distances. Numbers indicate percentage velocity perturbations. 
indicating lower-than-normal velocities. Note that all these models invariably show the presence of a high velocity region within the DVP surrounded by a relatively low velocity region. The magnitude and lateral extent of the velocity perturbations vary with the model. Although the anomalies are reflected in all the models presented below, the first one with a layer thickness of $40-100 \mathrm{~km}$ shows only a small variance improvement $(16 \%)$ compared with others whose variance improvement ranges from $26-32 \%$, suggesting that the high velocity anomaly (upto $4 \%$ ) is confined to a depth range of $100-400 \mathrm{~km}$ (table 3). To further constrain the anomalous domain we then analysed a large set of three-layer models. Two of these (with variance improvements $53 \%$ and $63 \%$ respectively) are shown in figure 10 , which show that the high velocity anomaly decreases both in magnitude and areal extent for the layer $0-100 \mathrm{~km}$ compared with that for the layer $0-150 \mathrm{~km}$. Significantly, the anomaly remains unaltered for depths of $400 \mathrm{~km}$. In order to further test that the anomalies exposed by the above models are not an artefact of the choice of the number of layers, we also tested a suite of 4 layered models for inverting the travel time residuals. Figure 11 and table 4 show the model with various layers lying between 0 and $40 \mathrm{~km}, 40$ and $200 \mathrm{~km}, 200$ and $400 \mathrm{~km}$ and $4(\%)$ and $600 \mathrm{~km}$ (variance improvement $46 \%$ ). These models show that the high velocity anomaly is confined to layers 2 and 3 in the depth range of $40-400 \mathrm{~km}$. As the depth to the top of the anomaly is reasonably well-constrained by the models (figure 10) described earlier we conclude that the high velocity anomaly coinciding with the southern two-thirds of the DVP exists in the depth range of $100-400 \mathrm{~km}$. The $400-600 \mathrm{~km}$ layer is characterized by the presence of a very diffused low velocity and is distinct from the overlying layers. Table 4 shows
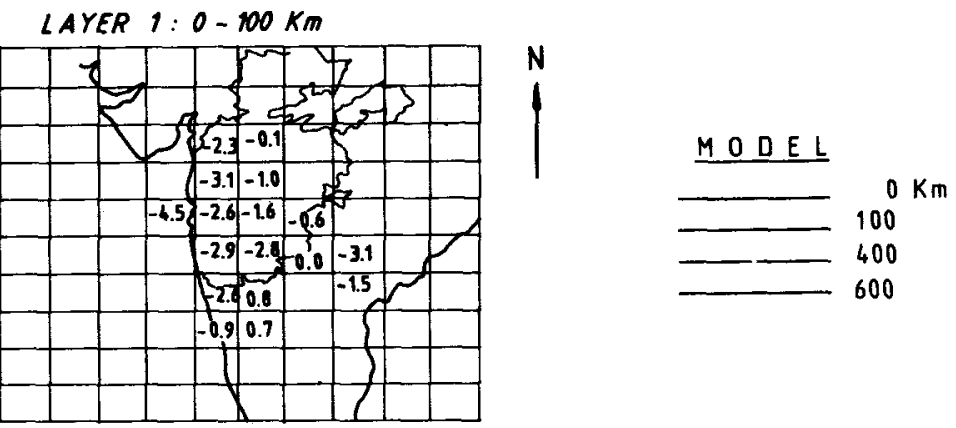

\section{$100 \mathrm{Km}$}

$\angle A Y E R \quad 2: 100-400 \mathrm{Km}$

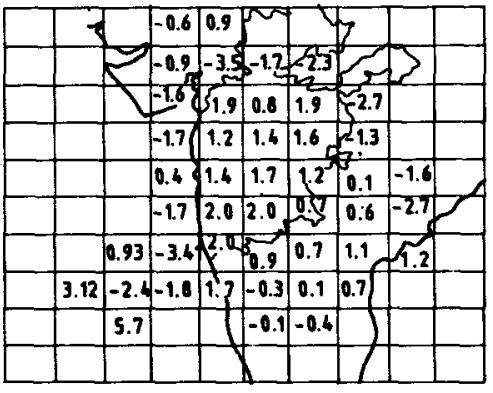

LAYER 3: $400-600 \mathrm{~km}$

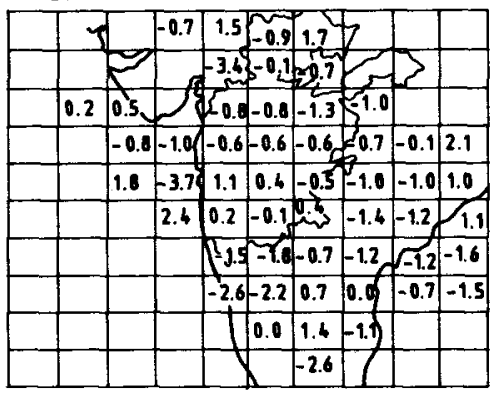

Figure 10a. Velocity perturbations in the three layers $(0-100,100-400,400-600 \mathrm{~km})$. 
LAYER 1: $0-150 \mathrm{Km}$

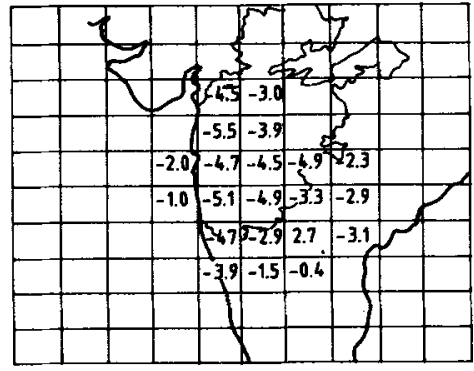

$400 \mathrm{Km}$

LAYER 2: $150-400 \mathrm{Km}$

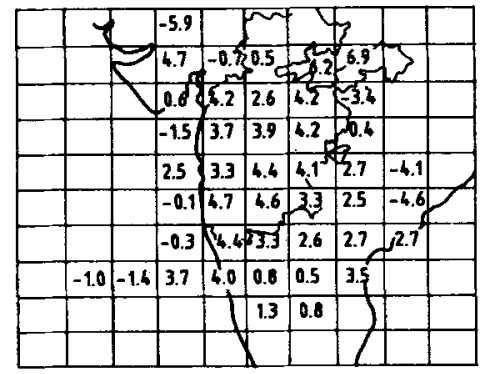

MODEL

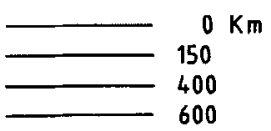

\section{$\mathrm{Km}$}

600
$N$
1

Figure 10b. Velocity perturbations in the three layers $(0-150,150-400,400-600 \mathrm{~km})$.

LAYER 2: $40-200 \mathrm{~km}$

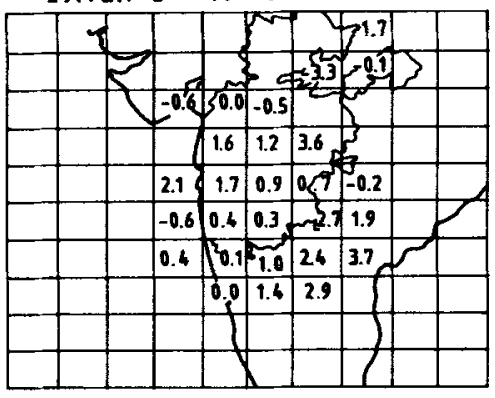

LAYER 3: $400-600 \mathrm{~km}$

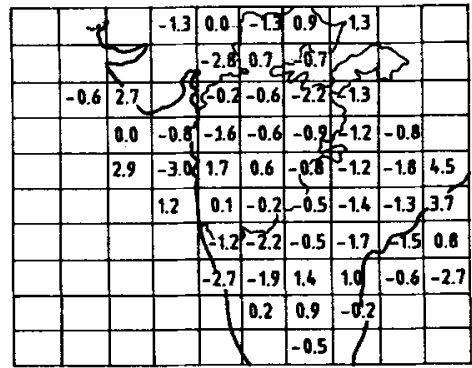




$$
\begin{aligned}
& \text { గ } 0 \text { \% } 0 \text { \% } 0 \text { i - } \\
& \approx m \pi n=\pi
\end{aligned}
$$

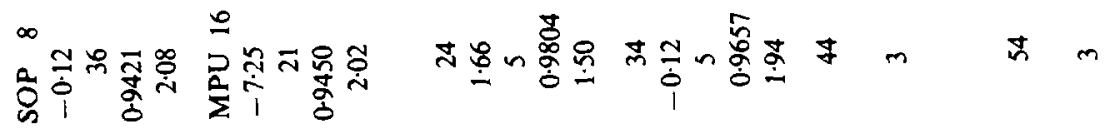

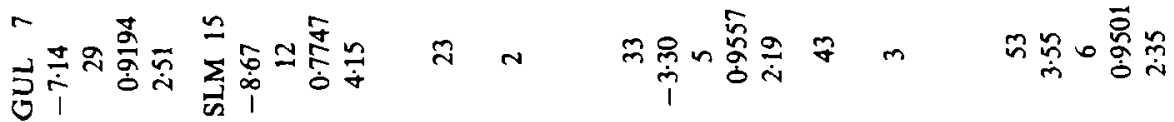

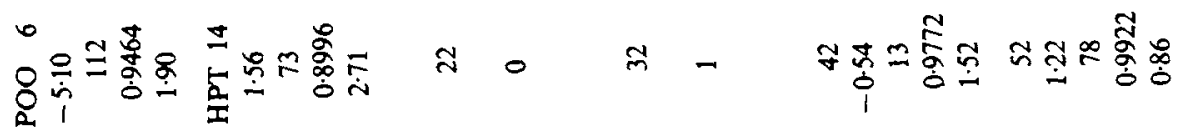

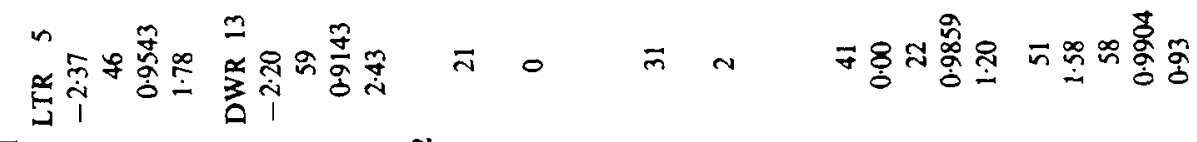

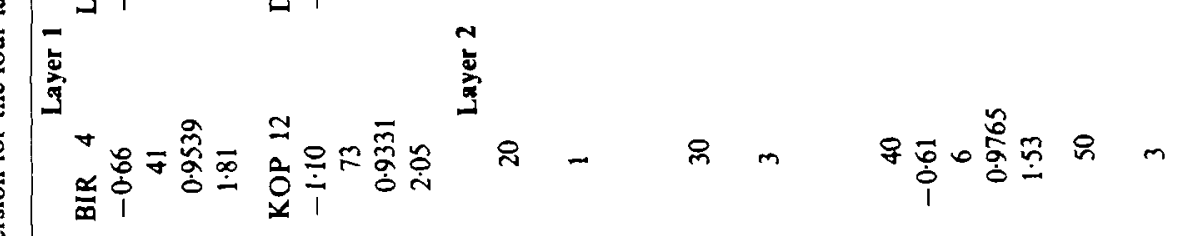

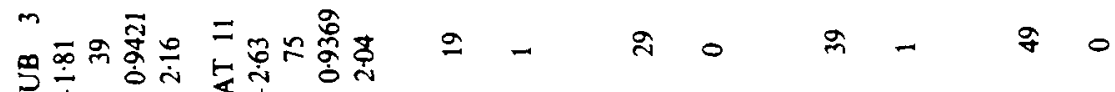

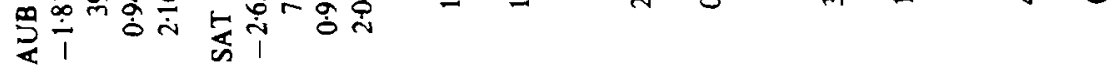

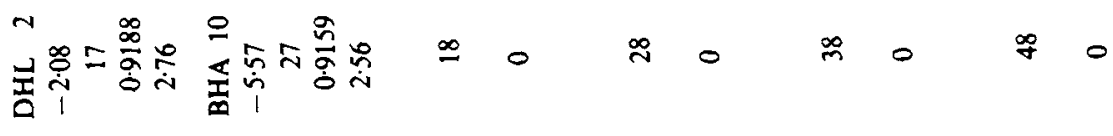
Z 
High velocity anomaly beneath Deccan volcanic province

51

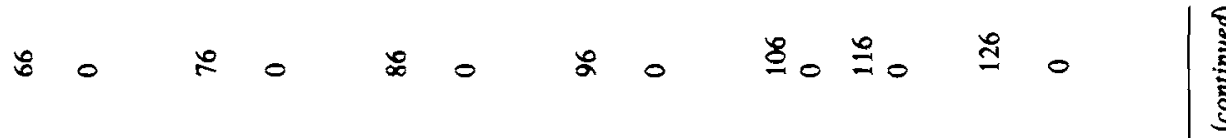

$$
\begin{aligned}
& \approx n \approx 0 \approx 0 \approx 0 \approx 000000
\end{aligned}
$$

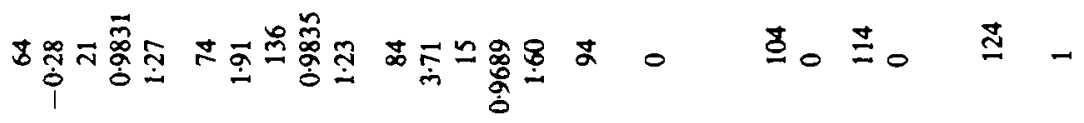

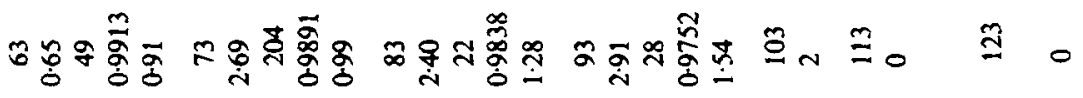

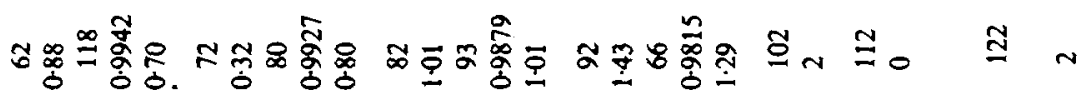

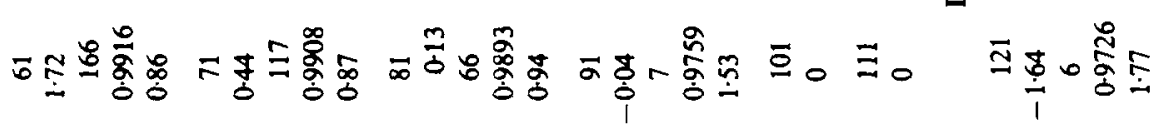

৪)

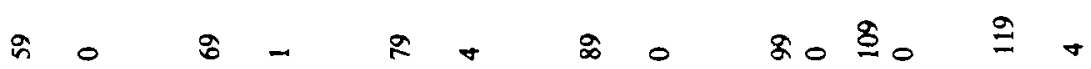

$\infty \quad \infty \quad 0 \quad \infty \quad \infty \quad \infty \quad \infty \quad \infty$

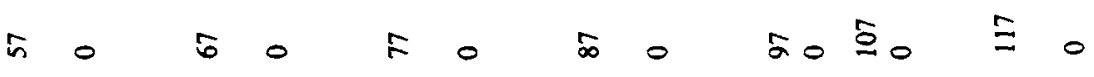




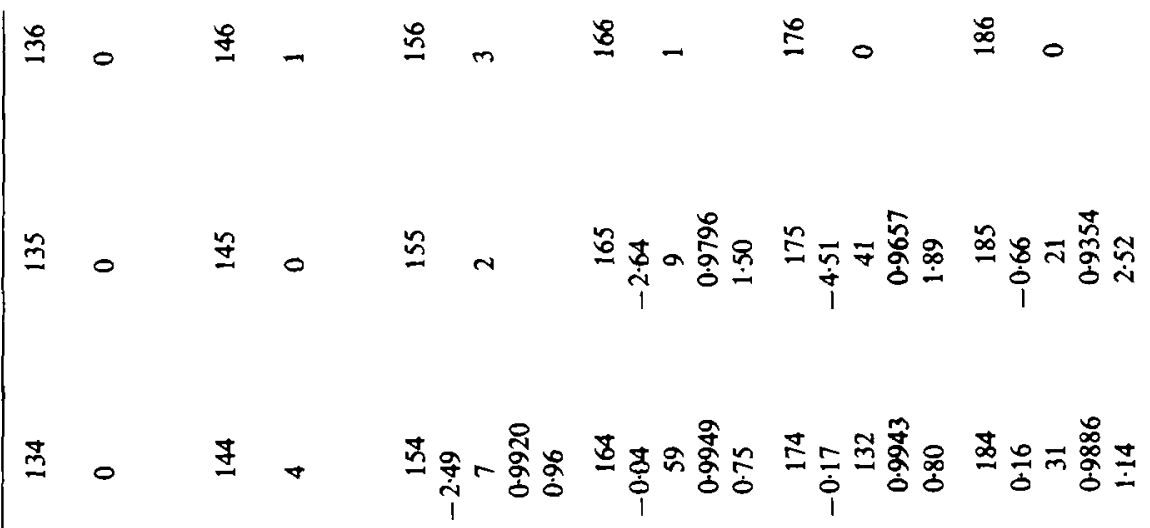

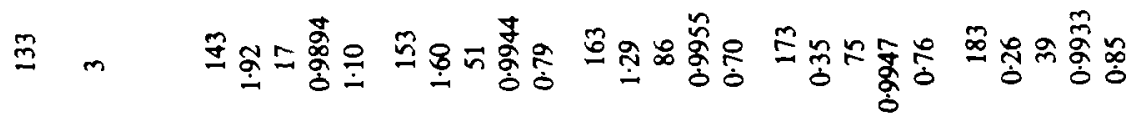

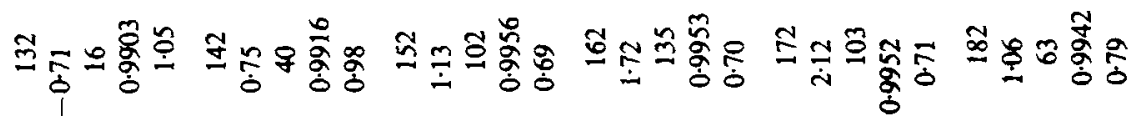

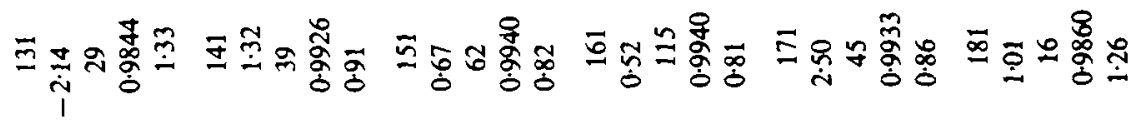

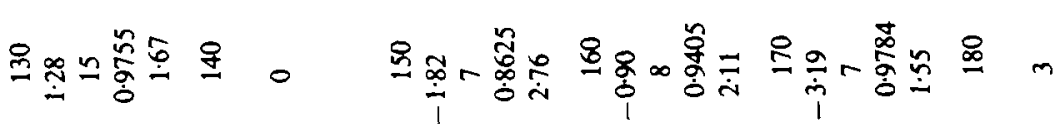

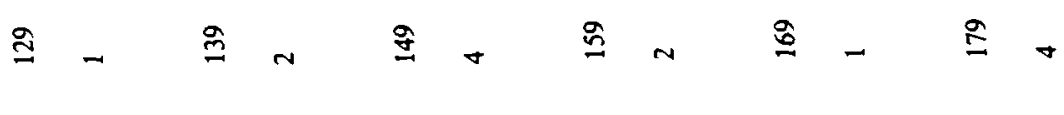

ฐ)

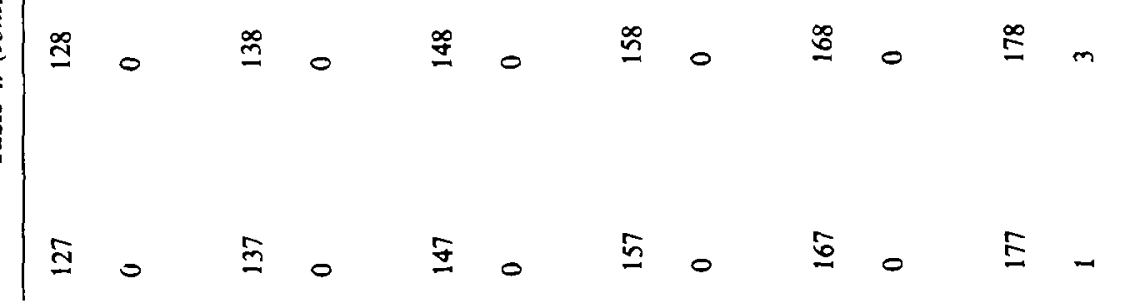




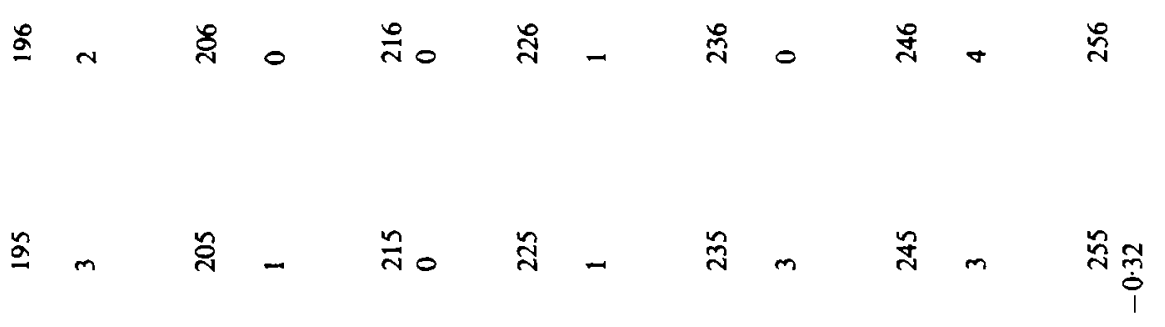

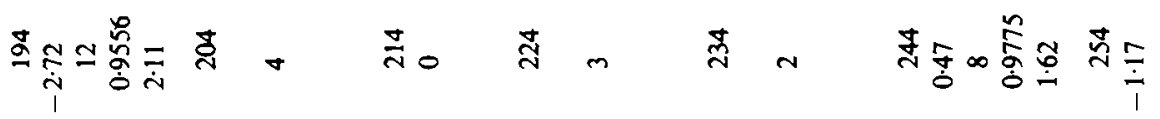

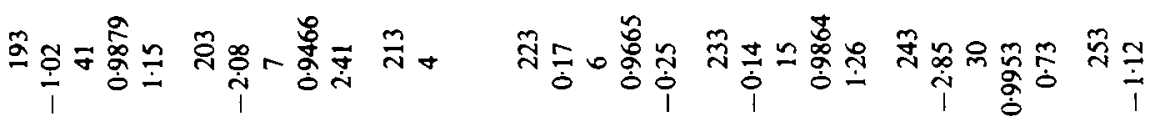

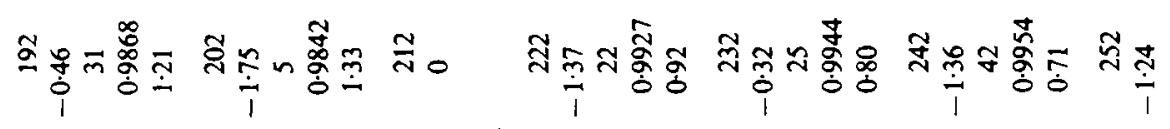
产

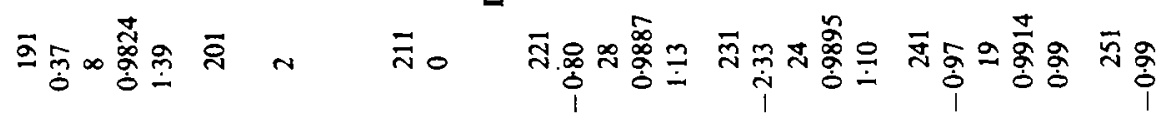

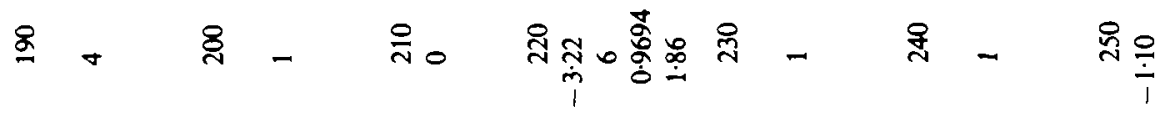

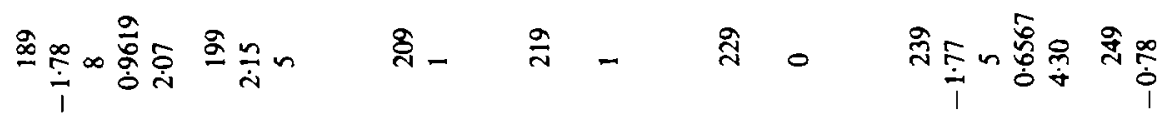

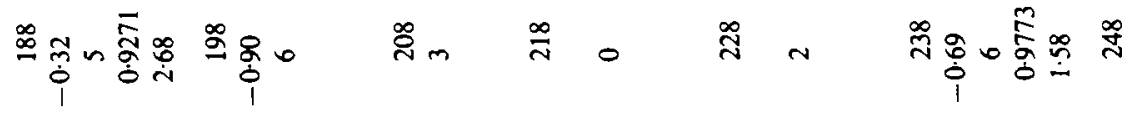

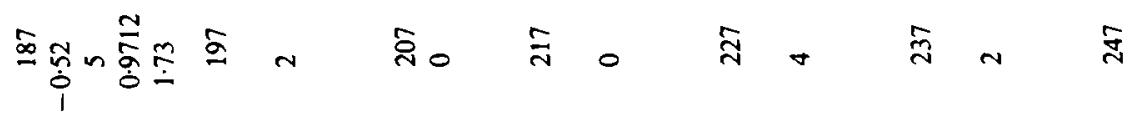




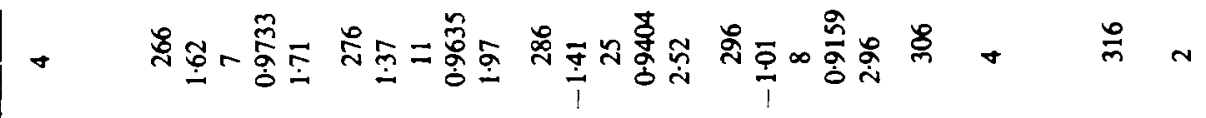

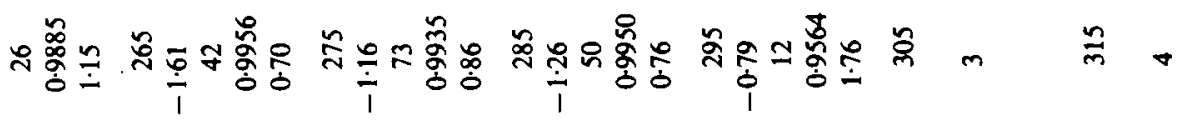

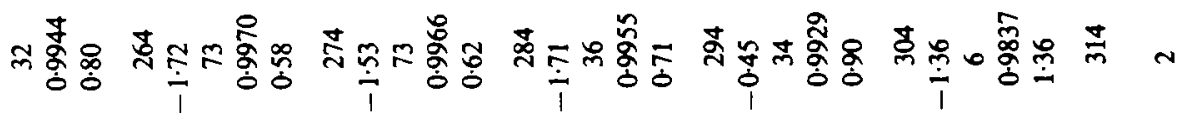
\&

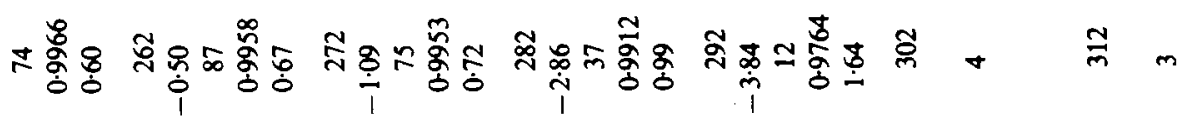

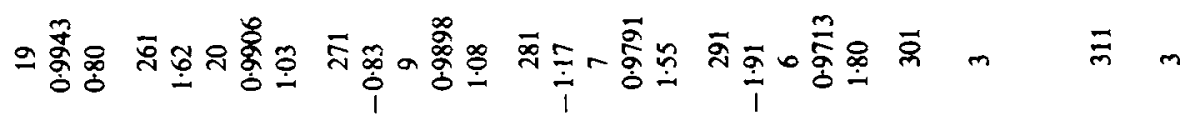

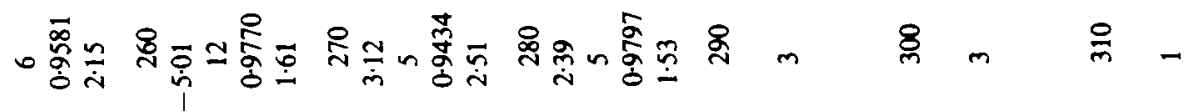

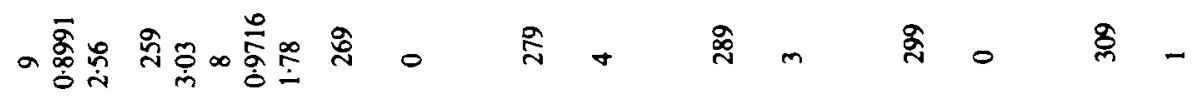

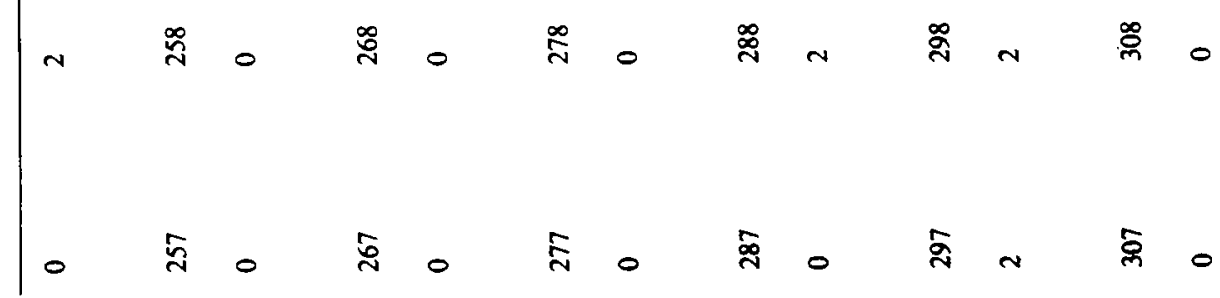


the diagonal elements of the resolution matrix along with the standard errors for this 4-layer model.

\section{Interpretation}

Existence of large-scale velocity anomalies in the upper mantle no longer evokes any surprise. Regional studies of one-dimensional velocity structure using travel-times, synthetic seismograms, and surface-wave dispersion, show that heterogeneous structure of the upper mantle is a rule rather than an exception in almost any region where observations are available. Teleseismic-residual studies show that velocity anomalies with dimensions of a few tens to a few hundreds of kilometers exist in the crust and upper mantle in North America and also in many other regions of the world (Aki 1982; Iyer 1984, 1988a, b; Thurber and Aki 1987; Iyer and Hitchcock 1988). In general, low-velocity anomalies are easier to interpret than high-velocity anomalies as the former seem to be generally associated with regions of young volcanism or recent orogeny. Some typical examples are: the Yellowstone hot spot (Iyer et al 1981); Rio Grande Rift (Parker et al 1984); Imperial Valley Spreading Centre (Humphreys et al 1984); Eastern U.S. (Taylor and Toksoz 1979); the Rhinegraben (Raikes and Bonjer 1983); and the Tarbela Area of Lesser Himalaya (Menke 1977). Higher-than-normal velocities, invariably found in active subduction zones and paleo-subduction zones are also reasonably well understood as they delineate subducting plates (for e.g., Japan: Hirahara 1981; Carapathian Arc: Romania: Oncescu et al 1984). Other high-velocity anomalies, not so well understood, are found in the upper mantle beneath the Baltic Shield (Husebye and Hovland 1982); Eastern Senegal, West Africa (Dorbath et al 1983); the Alps (Baer 1980); the North China Basin (Shedlock and Roecker 1987); the Timber Mountain Caldera Complex, Nevada (Evans and Oliver 1987); and the Southern Sierra Nevada, California (Humphreys et al 1984; Humphreys 1987), to name a few. Humphreys (1987) interprets the vertical, tongueshaped, high-velocity anomaly extending to depths of $300-400 \mathrm{~km}$ in Sierra Nevada, as representing a descending sub-crustal lithosphere caused by small-scale convection. Available case histories on large-scale high-velocity anomalies, though similar in size and velocity contrast, are simply too few to allow any detailed comparisons with the anomaly which we have delineated beneath the DVP.

A fundamental question to be addressed regarding the DVP anomaly is the following. Is the anomaly at all related to the Deccan volcanism or is it simply an integral part of the architecture of the upper mantle of the Indian shield? There is enough evidence from global and continental scale geophysical data to show that old continents are indeed far from being homogeneous in their deep crust and upper mantle structure. Our modelling results lead to startling revelations indicating the presence of an anomalously thick high-velocity region beneath the DVP. The presence of such an anomaly at depths much greater than the conventionally accepted plate thickness (of about $100-200 \mathrm{~km}$ ) could be best explained in the light of the concept of continental tectosphere (Jordan 1975, 1979a, b) and stabilization of cratons (Pollack 1986). The existence of lithospheric roots to depths as great as $400 \mathrm{~km}$ has been inferred earlier in Western North America, Superior Province of Canada and South Africa based on petrological, geothermal and seismological investigations (Grand and Helmberger 1984; Pollack 1986; Lam and Jordan 1987; Silver and Chan 1988). The 
tectosphere is proposed to be a region of massive basalt depletion and is predominantly composed of olivine and orthopyroxene, resulting in a density reduction of $1 \%$ and an increase in $S$-wave velocity due to lowering of the iron content. To constrain the density of the anomalous region beneath the DVP, we analysed two gravity profiles (west to east and north to south) taken from the $5 \mathrm{mgal}$ contour Bouguer gravity anomaly map of India. These indicate the presence of a long wavelength, $35 \mathrm{mgal}$ gravity low present over the region of high-velocity anomaly. A preliminary inversion of the data delineates the lower density region roughly lying within the high velocity anomalous region in the depth range of $100-300 \mathrm{~km}$, with a density contrast of about -0.006 to -0.013 grams per cc (D Srinagesh, personal communication).

The other line of evidence is from $S$-wave residuals at POO relative to HYB taken from ISC catalogues (figure 12). In spite of the poor quality of data and consequent high scatter, it is quite obvious that we see an azimuthal pattern broadly similar to that shown by $P$-wave residuals at $P O O$. The $S$-residuals are about three times as large as the corresponding $P$-wave residuals shown alongside for comparison. Shear wave structure beneath the DVP delineated by Montagner (1986) using surface wave data also reveals the existence of a high-velocity anomalous zone in the depth range of $85-250 \mathrm{~km}$. However, the continuation of this anomaly beyond $250 \mathrm{~km}$ depth is not observed due to data constraints in the surface wave study.

\section{Source of Deccan basalts}

Let us now consider the question of the source of Deccan basalts in the light of the existence of a deep continental root under this region, which must have acquired a coherent stable structure long before the Gondwanaland break-up considering that the craton came into existence earlier than at least 2.5 billion years. The formation of tectosphere due to the depletion of volatiles which elevates the solidus, renders the upper mantle region more resistant to subsequent melting. Such stable regions are observed to have normal shield heat flow values (Pollack 1986). This is in conformity with the observed normal heat flow over DVP (Gupta and Gaur 1984). The very presence of the tectosphere beneath the DVP would therefore inhibit the development of large scale perforations which are believed to have been responsible for the extrusion of such extensive flood basalts, implying the absence of their source region directly beneath the DVP. On the other hand, it is significant to note that in all the models, the per cent velocity anomaly beneath the westernmost part of the DVP in the depth range of $40-200 \mathrm{~km}$ is relatively less than that observed in other parts. This is also reflected in the results of surface wave study (Nataf et al 1984) showing a regional low shear wave velocity enveloping the entire Arabian Sea extending from the east coast of Africa to the west coat of India. However, it is significant to observe that the above low velocity zone is confined to the west of our westernmost profile 2 .

Also, recent studies of major and trace element abundances and inter-element ratios as well as $\mathrm{Sr}$ isotopic ratios in selected samples combined with extensive field checks in the western parts of the DVP show that the flow sections do not correspond to a classical layer cake-sequence. Instead, they appear to be comprised of a sequence of thickes and older formations in the north which become thinner in the south and are superceded by southward thickening of younger ones that taper out further southwards. This interlocking sequence strongly suggests that the flows are derived 


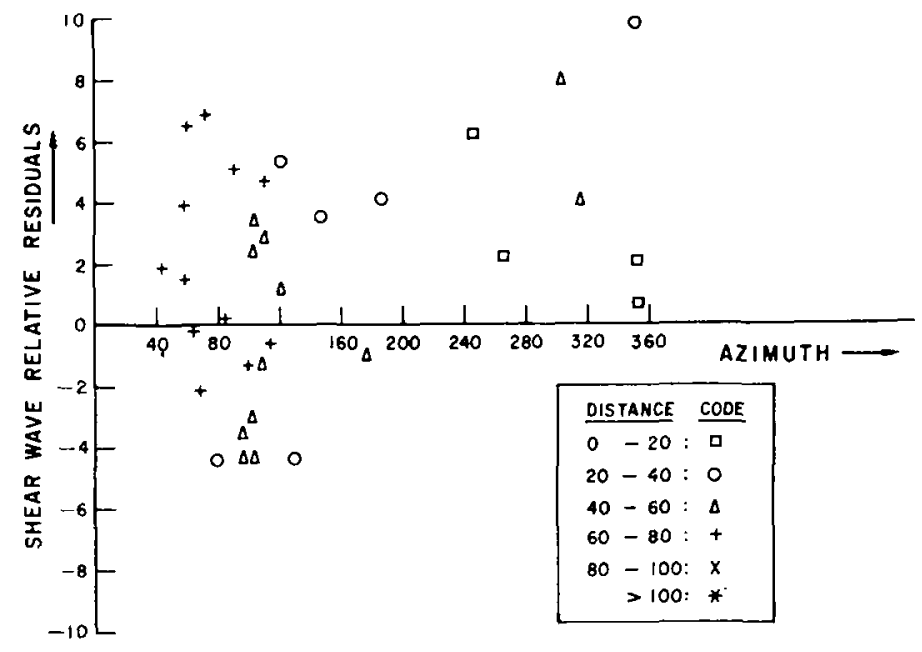

RELATIVE SHEAR WAVE TRAVEL TIME RESIDUALS

AT POONA WITH RESPECT TO HYDERABAD

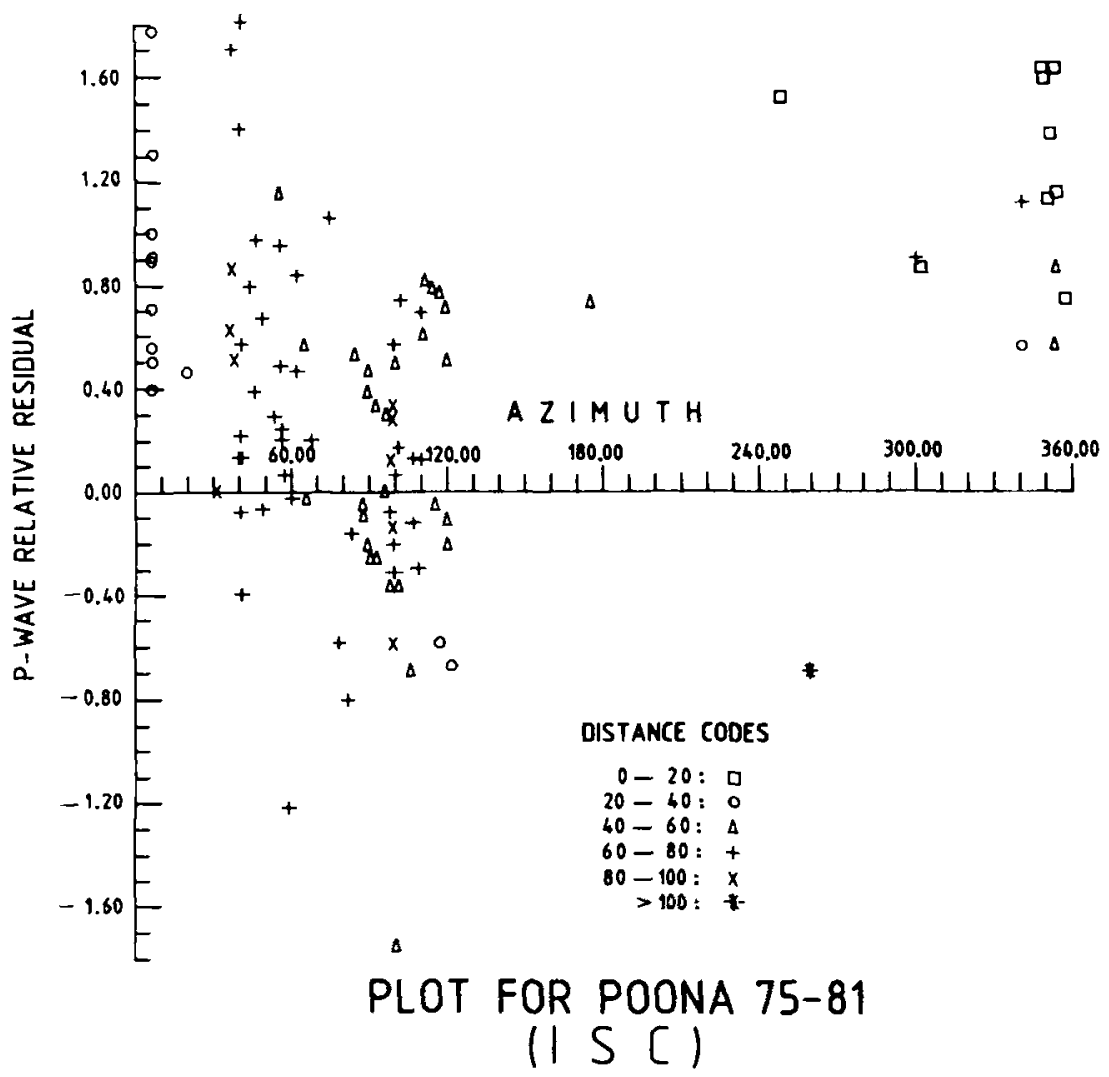

Figure 12. Typical azimuthal variation of $P$ and $S$-wave relative residuals at $P O O$ with respect to HYB compiled from the International Seismological Summary catalog. 
from southward migration of a centre of magmatic egress rather than from a system of fissures tapping the lower crust or upper mantle directly beneath the DVP. Further, $\mathrm{Nd}$ and $\mathrm{Sr}$ isotopic values of least contaminated Deccan Tholeiites from different areas of the DVP fall in a restricted field of high $\varepsilon_{N d}$ which closely approach those of old and present-day Central Indian ridge basalts. This strongly suggests that the Deccan basalts were derived from the same large region of isotopically homogeneous oceanic mantle (Mahoney 1988) which feeds the Central Indian ridge. Considering further that the bulk of Deccan basalts were formed about $65 \mathrm{Ma}$ ago shortly after the rifting between India and Madagascar $(80 \mathrm{Ma})$ when the ridge axis jumped towards India (Norton and Sclater 1979; Schlich 1982), a more natural channel for their outpouring would appear to be associated with the rift systems that eventually fashioned the Indian west coast passive continental margin. If the above visualization of the formation of Deccan basalts is correct, we should expect to see a low velocity zone (LVZ) on the western periphery of the DVP representing the source of the Deccan basalts perhaps characterized by high heat flow values but no thermal anomaly over the DVP. While the heat flow over the LVZ still remains to be measured, the other two predictions from the model are indeed confirmed by observational evidence.

\section{Conclusions}

Using teleseismic $\boldsymbol{P}$-wave residual tomography, we have identified a large region in the upper mantle beneath the Deccan volcanic province in which the $P$-wave velocity is higher than that in the surrounding region by 1 to $4 \%$. This high velocity anomaly exists coherently in the depth range of $100-400 \mathrm{~km}$. This implies that a coherent, colder and comparatively rigid lithospheric root extends to a depth of about $400 \mathrm{~km}$ beneath the DVP. The anomaly in the depth range of $400-600 \mathrm{~km}$ is distinctly weaker and appears to be decoupled from the overlying layers. This is as we expect owing to diminished ability of the asthenosphere to retain heterogeneities over extended periods of geologic times. Persistence of the anomaly in the upper mantle is however maintained through a stable geochemical reordering and formation of a tectosphere beneath the craton which together translate coherently over the underlying weaker mantle.

\section{Acknowledgements}

Support for this work came from three organizations: the Indo-US Subcommission on Education and Culture which awarded a fellowship to Iyer to spend 13 months in India; the National Geophysical Research Institute which provided full research support; and the U.S. Geological Survey which permitted Iyer's visit to India and completion of the research on his return to USA. Special thanks are due to the Director and staff of the Indian Institute of American Studies, New Delhi for efficiently managing Iyer's fellowship and assisting him in innumerable ways. We are greatly indebted to the various universities, colleges and many individuals for the warm cooperation extended to us in running our field stations during this work. We are very grateful to these organisations and also to many field assistants from NGRI, who participated in the experiment, to Phil Dawson for extensive support in data 
analysis and to Tim Hitchcock and $\mathbf{M}$ Jayarama Rao for help throughout the project including preparation of figures for this manuscript. Grateful thanks are also due to M/s. N K Venkatesh and G Ramakrishna Rao for the excellent typing of the manuscript.

\section{References}

Achauer U, Greene L, Evans J R and Iyer H M 1986 Nature of magma chamber underlying the Mono craters area, eastern California, as determined from teleseismic travel time residuals; J. Geophys. Res. 91 13872-13891

Aki K 1982 Three-dimensional seismic inhomogeneities in the lithosphere and asthenospehre: evidence for decoupling in the lithosphere and flow in the asthenosphere; Rev. Geophys. Space Phys. 20 161-170

Aki K, Christoffersson A and Husebeye E S 1927 Determination of the three-dimensional seismic structure of the lithosphere; J. Geophys. Res. 82 277-296

Baer M 1980 Relative travel time residuals for teleseismic events at the new Swiss seismic station network; Ann. Geophys. 36 119-126

Dorbath C, Dorbath L, Le page A and Gaulon R 1983 The West-African craton margin in eastern Senegal; a seismological study; Ann. Geophys. $125-36$

Ellsworth W L and Koyanagi R Y 1977 Three-dimensional crust and mantle structure of Kilanea Volcano, Hawaii; J. Geophys. Res. 82 5379-5394

Evans J R 1982 Compressional wave velocity structure of the upper $350 \mathrm{~km}$ under the eastern Snake River Plain near Rexburg. Idaho; J. Geophys. Res. 87 2654-2670

Evans J R and Oliver H W 1987 Comparison of Timber Mountain caldera complex, Nevada, with Yellowstone; speculations on mechanisms (Abs), Abstract Volume; Hawaii Symposium on How Volcanoes Work (Hilo, Hawaii: Hawaiian Volcano Observatory) p. 67

Grand S P and Helmberger D 1984 Upper mantle shear structure of North America; Geophys. J. R. Astron. Soc. $76399-438$

Gupta M L and Gaur V K 1984 Surface heat flow and probable evolution of Deccan volcanism; Tectonophysics 105 309-318

Hirahara K 1981 Three-dimensional seismic structure beneath southwest Japan: the subducting-phillippine sea plate; Tectonophysics 79 1-44

Herrin E 1968a Seismological tables for P; Seismol. Soc. Am. Bull. 58 1193-1241

Herrin E 1968b P-wave velocity distribution in the mantle; Seismol. Soc. Am. Bull. 58 1223-1225

Humphreys E 1987 Mantle dynamics of the southern Great Basin-Sierra Nevada region (Abs); EOS Trans. Am. Geophys Union 681450

Humphreys E and Clayton R W 1988 Adaptation of back-projection tomography to seismic travel time problems; J. Geophys. Res. 93 1073-1085

Humphreys E, Clayton R W and Hager B H 1984 A tomographic image of mantle structure beneath southern California; Geophys. Res. Lett. 11 625-627

Husebye ES and Hovland J 1982 On upper mantle seismic heterogeneities beneath Fennoscandia; Tectonophysics 90 1-17

Iyer H M 1984 Geophysical evidence for the locations, shapes and sizes, and internal structures of magma chambers beneath regions of Quaternary volcanism. In Relative contributions of mantle, oceanic crust, and continental crust to magma genesis, (eds) S Moorbath, R N Thompson and E R Oxburg (Philos Trans. R. Soc. London) A310 473-510

Iyer H M 1988a Seismic tomographys. Encyclopedia of geophysics (ed.) D James, (Van Nostrand Reinhold and $\mathrm{Co}$.) (in press)

Iyer H M $1988 b$ Seismological detection and delineation of magma chambers beneath intraplate volcanic centres in western USA. In Modelling volcanic processes (eds) C King and R Scaroa (Braunschweig/ Wiesbaden: Friedr. Vieweg and Sohn) pp. 1-56

Iyer H M, Evans J R, Zandt G, Stewart R M, Coakley J M and Roloff J N 1981 A deep low-velocity body under the Yellowsione caldera, Wyoming: delineation using teleseismic $P$-wave residuals and tectonic interpretation; Geol. Soc. Am. Bull. 92 Part I (Summary), 792-798; part II (full text in microfische edition) $1471-1646$

Iyer H M and Hitchcock T 1988 Upper mantle velocity structure in continental US and Canada. Geophysical framework of the Continental United States; Geol. Soc. Am. Mem. (in Press) 
Jordan T H 1975 The continental tectosphere; Rev. Geophys. Space Phys. 13 1-12

Jordan T H 1979a Lateral heterogeneity and mantle dynamics; Nature (London) 257 745-750

Jordan T H 1979b The deep structure of continents; Sci. Am. 240 92-107

Kaila K L 1982 Deep seismic sounding studies in India; Geophys. Res. Bull. NGRI, India (Special Issue) 20 309-328

Kaila K L, Tewari H C and Sarma, P L N 1981 Crustal structure from deep seismic sounding studies along Navibandar-Amreli profile in Saurashtra, India. In Deccan volcanism and related basalt provinces in other parts of the world (eds) K V Subba Rao and R N Sukheswala, Geol. Soc. India Memoir No. 3 pp. 218-232

Kaila K L, Reddy P R, Dixit M M and Koteswara Rao P 1985 Crustal structure across the Narmada-Son lineament, Central India from deep seismic soundings; J. Geol. Soc. India $26 \mathbf{4 6 5 - 4 8 0}$

Kailasam L N 1975 Epirogenic studies in India with reference to recent vertical movements; Tectonophysics 29 505-521

Lam A L L and Jordan T H 1987 How thick are the continents: J. Geophys. Res. 92 14007-14026

Mahoney J J 1988 Deccan basalts. In continental flood basalts (ed.) J D Macdougall (Kluiser, Norwell, MA)

Menke W H 1977 Lateral inhomogeneities in $P$ velocity under the Tarbela array of the lesser Himalayas of Pakistan; Bull. Seismol. Soc. Am. 67 725-734

Montagner J P 1986 Regional three-dimensional structures using long period surface waves; Ann. Geophys. $4283-294$

Morgan W J 1972 Deep mantle convection plumes and plate motions; Bull. Am. Assoc. Petrol Geol. $\mathbf{5 6}$ 203-213

Nataf H C, Nakanishi I and Anderson D L 1984 Anisotrophy and shear-velocity heterogeneities in the upper mantle; Geophys. Res. Lett. 11 109-112

Norton I O and Sclater J G 1979 A model for evolution of the Indian Ocean and break-up of Gondwanaland; J. Geophys. Res. 84 6803-6830

Oncescu M C, Burlacu V, Anghel M and Smalbergher V 1984 Three-dimensional p-wave velocity image under the Carpathinan Arc; Tectonophysics 106 305-319

Parker E C, Davis P M, Evans J R, Iyer H M and Olsen K H 1984 Upwarp of anomalous asthenosphere beneath the Rio qrande rift; Nature (London) 312 354-356

Pollack H N 1986 Cratonization and thermal evolution of the mantle; Earth. Planet. Sci. Lett., 80 175-182

Qureshy M N 1981 Gravity anomalies, isostasy and crust mantle relations in the Decan Trap and contiguous regions, India. In Deccan volcanism and related baslt provinces in other parts of the world (eds) K V Subba Rao and R N Sukheswala, Geol. Soc. India Memoir No. 3 185-197

Raikes S and Bonjer K P 1983 Large-scale mantle heterogeneity beneath the Rheinish Massif and its vicinity from teleseismic p-residuals measurements. In Plateau uplift (eds) $\mathrm{K}$ Fuchs et al (Berlin Heidelberg: Springer-Verlag) 315-331

Shedlock K M and Roecker S W 1987 Elastic wave velocity structure of the crust and upper mantle beneath the North China Basin; J. Geophys. Res. 92 9327-9350

Schlich R 1982 The Indian Ocean: Aseismic ridges, spreading centres, and oceanic basins. In The oceans hasins and margins, Vol. 6 The Indian Ocean (eds) A E M Nairn and F G Stelhi (New York: Plenum Press) $51-147$

Silver P G and Chan W W 1988 Implications for continental structure and evolution from seismic anisotropy; Nature (London) 335 34-39

Takin M 1966 An interpretation of the positive gravity anomaly over Bombay on the west coast of India; Geophys. J. R. Astron. Soc. 11 527-537

Taylor S R and Toksoz M N 1979 Three-dimensional crust and upper mantle structure of the northern United States; J. Geophys. Res. 84 7627-7644

Thurber C H and Aki K 1987 Three-dimensional seismic imaging, Ann. Rev. Earth Planet. Sci. 15 115-139 